\title{
ELIASHBERG'S PROOF OF CERF'S THEOREM
}

\author{
HANSJÖRG GEIGES AND KAI ZEHMISCH
}

\begin{abstract}
Following a line of reasoning suggested by Eliashberg, we prove Cerf's theorem that any diffeomorphism of the 3-sphere extends over the 4-ball. To this end we develop a moduli-theoretic version of Eliashberg's filling-withholomorphic-discs method.
\end{abstract}

\section{INTRODUCTION}

The abelian group $\Gamma_{n}$ of orientation preserving diffeomorphisms of the $(n-1)$ dimensional sphere $S^{n-1}$ modulo those that extend to a diffeomorphism of the $n$-ball $D^{n}$ plays an important role in differential topology, cf. [18. By the classical work of Kervaire-Milnor [17] on homotopy spheres and Smale's [29] solution of the higher-dimensional Poincaré conjecture, $\Gamma_{n}$ can be identified with the set of oriented smooth structures on the topological $n$-sphere for $n \geq 5$. The correspondence is given by associating with $[f] \in \Gamma_{n}$ the smooth structure on $S^{n}$ obtained by using the diffeomorphism $f$ of $S^{n-1}$ to glue two copies of $D^{n}$ along their boundary.

It is easy to see that $\Gamma_{1}$ and $\Gamma_{2}$ are trivial. The result $\Gamma_{3}=0$ is due independently to Munkres 24] and Smale 28. The argument of Munkres is quite elementary; using the Poincaré-Bendixon theorem for foliations of the plane, Smale actually proves the stronger statement that the 3 -dimensional orthogonal group is a strong deformation retract of the diffeomorphism group of the 2 -sphere. For $n \geq 5$, the groups $\Gamma_{n}$ are amenable to computation by the results of Kervaire-Milnor, for instance $\Gamma_{5}=\Gamma_{6}=0$, and $\Gamma_{7}$ is the cyclic group of order 28 .

The statement $\Gamma_{4}=0$ is known as Cerf's theorem [4. One consequence of this result is that there are no exotic smooth structures on $S^{4}$ that can be obtained by gluing two 4-discs. Thanks to the obstruction theory of Munkres [25] for smoothings of combinatorial manifolds, Cerf's theorem also implies, for instance, that every combinatorial $n$-manifold admits a smoothing for $n \leq 7$, unique up to diffeomorphism for $n \leq 6$.

In [6] Eliashberg proposed an ingenious proof of Cerf's theorem based on his classification of contact structures on $S^{3}$ and his method of filling with holomorphic discs [5]. Eliashberg-Polterovich [7] gave another topological application of this method; they showed that the space of local Lagrangian 2-knots in $\mathbb{R}^{4}$ is contractible.

Unfortunately, the survey paper [5] does not include proofs of the essential compactness and regularity properties of holomorphic discs. In the present paper we give a proof of Cerf's theorem along the lines suggested by Eliashberg. Rather than simply filling in the details, we develop an alternative approach to the filling with holomorphic discs in a moduli-theoretic framework, for which the technical foundations have been laid in the magisterial monograph of McDuff-Salamon 22 . 
Here is an outline of the paper. In Section 2 we describe the basic set-up of Eliashberg's proof, and then indicate where our strategy differs from Eliashberg's. In Section 3 we recall Eliashberg's argument why it suffices to prove the extension result for contactomorphisms of the standard contact structure $\xi$ on $S^{3}$.

In Section 4 we introduce the moduli space of holomorphic discs on which our proof of Cerf's theorem is built. The boundaries of the holomorphic discs in question are required to lie in a family of totally real submanifolds; in a different setting such varying boundary conditions have also been investigated by Wendl [30]. As described in Section 5, a suitable evaluation map on this moduli space then gives an 'explicit' extension of a given contactomorphism of $\left(S^{3}, \xi\right)$ to a diffeomorphism of $D^{4}$. Sections 6 and 7 are devoted to establishing compactness and transversality results for our moduli space.

In order to ascertain that the holomorphic discs in question are embedded, we need a relative adjunction inequality, which in turn relies on results about positivity of intersection. These are demonstrated in Section 9 , preceded by an account of the topological intersection theory of discs in Section 8 ,

One important word about notation: following [22, and with apologies to hyperbolic geometers, we write $\mathbb{D} \subset \mathbb{C}$ and $\mathbb{H} \subset \mathbb{C}$ for the closed unit disc and upper half-plane, respectively.

\section{IDEA OF THE PROOF}

Regard the 3-sphere $S^{3}$ as the unit sphere in $\mathbb{C}^{2}$ with complex Cartesian coordinates $\left(z_{1}=x_{1}+i y_{1}, z_{2}=x_{2}+i y_{2}\right)$. Let $H$ be the height function on $S^{3}$ given by projection onto the $y_{2}$-coordinate. For $t \in(-1,1)$ the level sets $S^{t}:=H^{-1}(t)$ define a smooth foliation of $S^{3} \backslash\{(0,0,0, \pm 1)\}$ by 2 -spheres. We regard the points

$$
q_{ \pm}^{t}:=\left(0,0, \pm \sqrt{1-t^{2}}, t\right)
$$

as the poles of these 2 -spheres.

This family of poles, together with the two poles $(0,0,0, \pm 1)$ of $S^{3}$, forms an unknot

$$
K:=\left\{\left(0,0, \pm \sqrt{1-t^{2}}, t\right): t \in[-1,1]\right\}
$$

in $S^{3}$. The complement $S^{3} \backslash K$ is foliated by circles that bound holomorphic discs

$$
D_{s}^{t}:=D^{4} \cap\left(\mathbb{C} \times\left\{x_{2}=s, y_{2}=t\right\}\right),|t|<1,|s|<\sqrt{1-t^{2}} .
$$

For each $t \in(-1,1)$, the circles $\partial D_{s}^{t}$ foliate the punctured 2-sphere $S^{t} \backslash\left\{q_{ \pm}^{t}\right\}$; we write $\mathfrak{C}_{t}$ for this foliation by circles.

Now let $\varphi$ be an orientation preserving diffeomorphism of $S^{3}$ that we wish to extend over $D^{4}$. Any isotopy from $\varphi$ to a diffeomorphism $\psi$ can be swept out over a collar neighbourhood of $S^{3}$ in $D^{4}$, where the collar coordinate serves as isotopy parameter. Thus, if $\psi$ extends over $D^{4}$, then so does $\varphi$. In other words, it suffices to find an extension for a suitably well-behaved representative in the isotopy class of $\varphi$.

By the disc theorem [12, Theorem 8.3.1], any orientation preserving diffeomorphism of an $n$-manifold is isotopic to one that fixes any given embedded $n$-disc. So we may require without loss of generality that $\varphi$ fix a neighbourhood of the unknot $K$.

Write $\tilde{S}^{t}:=\varphi\left(S^{t}\right)$ for the images of the 2 -spheres $S^{t}$ under $\varphi$. Then $\varphi\left(\mathfrak{C}_{t}\right)$ is a foliation of the punctured 2-sphere $\tilde{S}^{t} \backslash\left\{q_{ \pm}^{t}\right\}$ by circles. Now the key idea is as 
follows. Suppose we were able to find a foliation of $D^{4} \backslash K$ by holomorphic discs with these properties:

(1) The boundaries of the discs foliate the punctured 2-spheres $\tilde{S}^{t} \backslash\left\{q_{ \pm}^{t}\right\}$.

(2) This foliation of $\tilde{S}^{t} \backslash\left\{q_{ \pm}^{t}\right\}$ can be deformed to $\varphi\left(\mathfrak{C}_{t}\right)$.

Then the proof of Cerf's theorem $\Gamma_{4}=0$ would reduce to $\Gamma_{2}=0$, or more precisely the parametric version of the latter, which says that the restriction map $\operatorname{Diff}\left(D^{2}\right) \rightarrow \operatorname{Diff}\left(S^{1}\right)$ is a Serre fibration with contractible fibre.

As observed by Eliashberg, this plan is feasible, provided that $\varphi$ is a contactomorphism of the standard contact structure $\xi$ on $S^{3}$. Then both $\varphi\left(\mathfrak{C}_{t}\right)$ and the foliation of $\tilde{S}^{t} \backslash\left\{q_{ \pm}^{t}\right\}$ given by the boundaries of holomorphic discs are transverse to the characteristic foliation $\tilde{S}_{\xi}^{t}$, so one foliation can be deformed into the other by an isotopy along the characteristic foliation. Moreover, Eliashberg's classification of contact structures on $S^{3}$ shows that any diffeomorphism of $S^{3}$ is indeed isotopic to a contactomorphism of $\left(S^{3}, \xi\right)$ - for the convenience of the reader we reproduce the proof of this fact in Section 3-, so this assumption on $\varphi$ is not restrictive.

In the present paper, Eliashberg's programme is carried out rigorously. However, we deviate from his scheme in one important respect. We define a moduli space of holomorphic discs in $D^{4} \subset \mathbb{C}^{2}$ whose boundaries lie on the punctured spheres $\tilde{S}^{t} \backslash\left\{q_{ \pm}^{t}\right\}$. The extension of the contactomorphism $\varphi$ over $D^{4}$ is then defined in terms of an evaluation map on this moduli space. That way we obtain easier control over the smoothness of the extension. This is true in particular near the singular set $K$, where the behaviour of the diffeomorphism is clear from the observation that the filling with holomorphic discs adapted to $\varphi$ coincides with the standard filling near $K$.

The reader will notice that the vanishing of $\Gamma_{2}$ is not mentioned explicitly in our proof. This is a consequence of our working with holomorphic discs with three marked points on the boundary. Such discs come with a fixed parametrisation, so we need no longer worry about choices of diffeomorphisms of $S^{1}$ and their extensions to $D^{2}$.

In the following subsections we describe the backcloth of our proof, mostly to set up notation.

2.1. Contact geometry of $S^{3}$. Consider the differential 1-form

$$
\lambda:=\frac{1}{2}\left(x_{1} d y_{1}-y_{1} d x_{1}+x_{2} d y_{2}-y_{2} d x_{2}\right)
$$

on $\mathbb{R}^{4}$. The 1-form $\alpha:=\left.\lambda\right|_{T S^{3}}$ on $S^{3}$ gives rise to a volume form $\alpha \wedge d \alpha$ defining the positive orientation of $S^{3}$ (with $S^{3}$ oriented as boundary of $D^{4} \subset \mathbb{C}^{2}$ ), so $\alpha$ is a contact form. Its kernel $\xi:=\operatorname{ker} \alpha$ is the (positive) standard contact structure on $S^{3}$. The 2 -form $\omega:=d \lambda$ is the standard symplectic form on $\mathbb{R}^{4}$.

The characteristic foliation $S_{\xi}^{t}$ induced by $\xi$ on the 2 -sphere $S^{t}$ is the singular 1-dimensional foliation defined by the intersection $T_{p} S^{t} \cap \xi_{p}$ for $p \in S^{t}$, with singular points where $T_{p} S^{t}$ and the contact plane $\xi_{p}$ coincide, which happens exactly at $p=q_{ \pm}^{t}$. Notice that the unknot $K$ made up of these singular points and the two poles of $S^{3}$ is transverse to $\xi$.

A contactomorphism of $\xi=\operatorname{ker} \alpha$ is a diffeomorphism $\varphi$ of $S^{3}$ such that $\varphi^{*} \alpha=f \alpha$ for some smooth function $f: S^{3} \rightarrow \mathbb{R}^{+}$. 
In Section 3 below we also have to use the notion of a tight contact structure, and we appeal to Gray stability of contact structures. Both concepts are explained in 10 .

2.2. Strict pseudoconvexity of $S^{3}$. Write $J_{0}$ for the standard complex bundle structure on $T \mathbb{C}^{2}$ induced by multiplication with $i$. The 3 -sphere $S^{3}$ may be regarded as the level set $\rho^{-1}(1)$ of the strictly plurisubharmonic function

$$
\rho: \mathbb{C}^{2} \longrightarrow \mathbb{R}, \quad\left(z_{1}, z_{2}\right) \longmapsto\left|z_{1}\right|^{2}+\left|z_{2}\right|^{2} .
$$

This implies that the complex tangencies $T_{p} S^{3} \cap J_{0}\left(T_{p} S^{3}\right)$ define a contact structure on $S^{3}$, given as the kernel of the contact form $-\left.(1 / 2) d \rho \circ J_{0}\right|_{T S^{3}}$, cf. [10, Chapter 5]. As is well known (and easy to check), this contact form equals the previously defined $\alpha$.

The essential consequence of $S^{3}$ being strictly pseudoconvex is that non-constant holomorphic discs in $\mathbb{C}^{2}$ with boundary on $S^{3}$ have their interior in the interior of $D^{4}$, see Proposition 4.2 for the precise formulation of this maximum principle.

The characteristic foliation $S_{\xi}^{t}$ being non-singular away from $q_{ \pm}^{t}$ is equivalent to $S^{t}$ not having any complex tangencies except at these two poles. In other words, $S^{t} \backslash\left\{q_{ \pm}^{t}\right\}$ is a totally real surface.

2.3. A holomorphic filling of $S^{3}$. For $|t|<1$ and $|s|<\sqrt{1-t^{2}}$ define a smooth real-valued function

$$
\theta(s, t):=\frac{t}{2 \sqrt{1-t^{2}}} \cdot \ln \left(\frac{\sqrt{1-t^{2}}+s}{\sqrt{1-t^{2}}-s}\right) .
$$

For each $t$ this defines a diffeomorphism from $\left(-\sqrt{1-t^{2}}, \sqrt{1-t^{2}}\right)$ to $\mathbb{R}$. Now consider the parametrisations

$$
u_{s}^{t}(z):=\left(\sqrt{1-s^{2}-t^{2}} \cdot e^{i \theta(s, t)} \cdot z, s, t\right), \quad z \in \mathbb{D}
$$

of the holomorphic discs $D_{s}^{t}$. The rotation factor $e^{i \theta(s, t)}$ has been chosen in such a way that each leaf of the characteristic foliation $S_{\xi}^{t}$ (outside $q_{ \pm}^{t}$ ) is parametrised by a map $s \mapsto u_{s}^{t}(z),|s|<\sqrt{1-t^{2}}$, for some $z \in \partial \mathbb{D}$. For this one simply needs to verify that the tangent vector $\partial_{s} u_{s}^{t}(z)$ to $S^{3}$ (for $z \in \partial \mathbb{D}$ ) lies in the kernel of the 1 -form $x_{1} d y_{1}-y_{1} d x_{1}-t d x_{2}$, which is the pull-back of $\alpha$ to $S^{t}$. Putting all these parametrisations together, we obtain a diffeomorphism

$$
\begin{array}{ccc}
F_{\mathrm{st}}: \quad(\mathbb{D} \times \operatorname{Int} \mathbb{D}, \partial \mathbb{D} \times \operatorname{Int} \mathbb{D}) & \longrightarrow & \left(D^{4} \backslash K, S^{3} \backslash K\right) \\
(z, s, t) & \longmapsto & u_{s}^{t}(z) .
\end{array}
$$

We can extend this map continuously over the boundary $\mathbb{D} \times \partial \mathbb{D}$ by sending $(z, s, t)$ with $|t|<1$ and $s= \pm \sqrt{1-t^{2}}$ to $q_{ \pm}^{t}$, and $(z, 0, \pm 1)$ to $(0,0,0, \pm 1)$. So the boundary is mapped onto $K$.

This map $F_{\text {st }}$ will be our prototype of a holomorphic filling. The formal definition of such fillings will be given in Section 5 .

2.4. Symplectic energy. The symplectic energy of the holomorphic disc $u_{s}^{t}$ is defined as $E\left(u_{s}^{t}\right)=\int_{\mathbb{D}}\left(u_{s}^{t}\right)^{*} \omega$. One computes

$$
E\left(u_{s}^{t}\right)=\pi\left(1-s^{2}-t^{2}\right) \leq \pi\left(1-t^{2}\right),
$$

i.e. for each $t$ we have a uniform bound on the energy. A general result to this effect will be proved in Proposition 4.7 
Writing $z$ in polar coordinates as $z=r e^{i \theta}$, we compute

$$
\alpha\left(\partial_{\theta} u_{s}^{t}(z)\right)=\left(1-s^{2}-t^{2}\right) / 2 \text { for } z \in \partial \mathbb{D} .
$$

This gives another way to verify the computation of the energy, since $\int_{\mathbb{D}}\left(u_{s}^{t}\right)^{*} \omega=$ $\int_{\partial \mathbb{D}}\left(u_{s}^{t}\right)^{*} \alpha$ by Stokes's theorem.

Since $\alpha$ evaluates positively on $\partial_{\theta} u_{s}^{t}(z)$ for $z \in \partial \mathbb{D}$, the curve $\left.u_{s}^{t}\right|_{\partial \mathbb{D}}$ is positively transverse to the characteristic foliation $S_{\xi}^{t}$. It is another consequence of the maximum principle that this holds true for arbitrary holomorphic discs, see Proposition 4.2

2.5. Bundle pairs and Maslov index. The holomorphic disc $u_{s}^{t}$ has boundary on the totally real submanifold $S^{t} \backslash\left\{q_{ \pm}^{t}\right\}$ of $\mathbb{C}^{2}$. So it defines a bundle pair in the sense of [22, Definition C.3.4], that is, a complex vector bundle $\left(u_{s}^{t}\right)^{*} T \mathbb{C}^{2}$ over $\mathbb{D}$ (here: the trivial $\mathbb{C}^{2}$-bundle) and a totally real subbundle $\left(\left.u_{s}^{t}\right|_{\partial \mathbb{D}}\right)^{*} T S^{t}$ over the boundary $\partial \mathbb{D}$. At the point $u_{0}^{t}\left(e^{i \theta}\right) \in \partial D_{0}^{t}$, the fibre of this totally real subbundle is given by $\mathbb{R} i e^{i \theta} \oplus \mathbb{R}$. From the axiomatic definition of the Maslov index $\mu$ given in [22, Theorem C.3.5] it follows that $\mu\left(u_{s}^{t}\right)=2$ for all $t \in(-1,1)$ and $|s|<\sqrt{1-t^{2}}$.

2.6. The standard neighbourhood of $K$. To avoid problems at the singular points $q_{ \pm}^{t}$, we design a set-up where the holomorphic filling of $D^{4}$ corresponding to some contactomorphism of $S^{3}$ coincides with the standard filling $F_{\text {st }}$ near $K$. For $\delta \in(0,1)$, we define a neighbourhood of $K$ by

$$
\mathcal{U}^{\delta}:=F_{\text {st }}\left(S^{1} \times\left\{1-\delta<s^{2}+t^{2} \leq 1\right\}\right) \subset S^{3} .
$$

The boundary $\partial \mathcal{U}^{\delta}$ is a Lagrangian torus with a holomorphic filling given by the restriction of $F_{\text {st }}$ to $\mathbb{D} \times\left\{s^{2}+t^{2}=1-\delta\right\}$. We shall require the contactomorphism of $\left(S^{3}, \xi\right)$ to act as the identity on some $\mathcal{U}^{\delta}$.

2.7. The 2-discs $Q_{k}$. In order to obtain a compact moduli space of holomorphic discs, one needs to quotient out the 3-dimensional automorphism group of $\mathbb{D}$ or, alternatively, place a restriction on three marked points in $\mathbb{D}$. We shall take the latter approach, using the three points $i^{k} \in \partial \mathbb{D}, k=0,1,2$ as markers.

We define three open 2-discs in $S^{3}$ by

$$
Q_{k}:=F_{\text {st }}\left(\left\{i^{k}\right\} \times \operatorname{Int} \mathbb{D}\right) \subset S^{3} .
$$

These discs are transverse to the punctured 2-spheres $S^{t} \backslash\left\{q_{ \pm}^{t}\right\}$, and the intersection is given by one leaf $\ell_{k}^{t}$ of the characteristic foliation $S_{\xi}^{t}$.

Notice that a contactomorphism $\varphi$ of $\left(S^{3}, \xi\right)$ will map the characteristic foliation $S_{\xi}^{t}$ to the characteristic foliation of the image sphere $\tilde{S}^{t}=\varphi\left(S^{t}\right)$. The discs $Q_{k}$ will be used in Lemma 7.2 to show that, as expected, imposing the condition that our holomorphic discs map $i^{k}$ into the leaf $\tilde{\ell}_{k}^{t}:=\varphi\left(\ell_{k}^{t}\right)$ will cut down the dimensions of the moduli space by 3 .

We also consider the smaller closed disc

$$
Q^{\delta}:=F_{\text {st }}\left(\{1\} \times\left\{s^{2}+t^{2} \leq 1-\delta\right\}\right) \subset Q_{0} .
$$

In Proposition 5.1 we shall set up a diffeomorphism between the $\operatorname{disc} \varphi\left(Q^{\delta}\right)$ and the moduli space of holomorphic discs adapted to $\varphi$, with three marked points and boundary outside the neighbourhood $\mathcal{U}^{\delta}$. 


\section{Reduction to CONTACT GEOMETRY}

In order to construct an extension of a given diffeomorphism of $S^{3}$ to a diffeomorphism of $D^{4}$, we first want to isotope it to a diffeomorphism adapted to the set-up of the previous section.

Proposition 3.1. Any orientation preserving diffeomorphism of $S^{3}$ is isotopic to a contactomorphism of $\left(S^{3}, \xi\right)$ that fixes an open neighbourhood $\mathcal{U}$ of $K$ pointwise.

Proof. Let $\varphi_{0}$ be a given orientation preserving diffeomorphism of $S^{3}$. Then $T \varphi(\xi)$ is a positive tight contact structure on the 3-sphere. By the uniqueness of such structures up to isotopy, and with Gray stability, we can isotope $\varphi_{0}$ to a contactomorphism $\varphi_{1}$ of $\xi$, cf. [10, Lemma 4.11.1].

There is a contactomorphism

$$
\left(\mathbb{R}^{3}, \operatorname{ker}(d w+u d v-v d u)\right) \longrightarrow\left(S^{3} \backslash\{(0,1,0,0)\}, \xi\right)
$$

which sends the unit circle $C$ in the $u v$-plane to $K$. An explicit description of such a contactomorphism can be found in [10, Proposition 2.1.8]; simply compose the contactomorphism

$$
\left(\mathbb{R}^{3}, \operatorname{ker}(d w+u d v-v d u)\right) \longrightarrow\left(S^{3} \backslash\{(0,0,0,1)\}, \xi\right)
$$

described there with the contactomorphism of $\left(S^{3}, \xi\right)$ induced by $\left(z_{1}, z_{2}\right) \mapsto\left(z_{2}, z_{1}\right)$.

This contactomorphism restricts to a contact embedding $j$ of a ball $B$ of radius 2 , say, in $\left(\mathbb{R}^{3}, \operatorname{ker}(d w+u d v-v d u)\right)$ into $\left(S^{3}, \xi\right)$, sending $C$ to $K$. A second contact embedding of this ball is given by $\varphi_{1} \circ j$. By the contact disc theorem [10, Theorem 2.6.7 and Remark 2.6.8], there is a contact isotopy whose time-1 map $\psi_{1}$ is a contactomorphism of $\left(S^{3}, \xi\right)$ such that $\psi_{1} \circ \varphi_{1} \circ j=j$.

So our initial diffeomorphism $\varphi_{0}$ is isotopic to the contactomorphism $\psi_{1} \circ \varphi_{1}$, which fixes the neighbourhood $\mathcal{U}:=j(\operatorname{int} B)$.

The isotopy from $\varphi_{0}$ to $\psi_{1} \circ \varphi_{1}$ can be swept out over a collar neighbourhood of $S^{3}=\partial D^{4}$ in $D^{4}$. Hence, Cerf's theorem will follow if we can find an extension of $\psi_{1} \circ \varphi_{1}$ to a diffeomorphism of $D^{4}$.

So from now on we consider a contactomorphism $\varphi$ of $\left(S^{3}, \xi\right)$ that fixes an open neighbourhood $\mathcal{U}$ of $K$ pointwise, and we choose $\delta \in(0,1)$ such that the closure $\overline{\mathcal{U}}^{\delta}$ of the standard neighbourhood $\mathcal{U}^{\delta}$ is contained in $\mathcal{U}$.

\section{BISHOP DISCS}

In Section 2 we described a simple filling of the 3-sphere by holomorphic discs, i.e. a foliation of $D^{4} \backslash K$ by discs with boundary in $S^{3} \backslash K$. We now want to construct another such filling, one that is related to the contactomorphism $\varphi$.

We begin by introducing the corresponding boundary value problem. For fixed $t \in(-1,1)$ we are looking for smooth (up to the boundary) solutions

$$
u^{t}:(\mathbb{D}, \partial \mathbb{D}) \longrightarrow\left(D^{4}, \tilde{S}^{t} \backslash\left\{q_{ \pm}^{t}\right\}\right)
$$

of the homogeneous Cauchy-Riemann equation $\bar{\partial} u^{t}=0$ (in other words: holomorphic discs) having boundary values in $\tilde{S}^{t}:=\varphi\left(S^{t}\right)$. We remarked before that the punctured 2-spheres $S^{t} \backslash\left\{q_{ \pm}^{t}\right\}$ are totally real submanifolds of $\mathbb{C}^{2}$. Hence, so are their images $\tilde{S}^{t} \backslash\left\{q_{ \pm}^{t}\right\}$ under $\varphi$, since $\varphi$ preserves the contact structure $\xi=T S^{3} \cap J_{0}\left(T S^{3}\right)$. 
In addition, we require the relative homotopy class $\left[u^{t}\right] \in \pi_{2}\left(D^{4}, \tilde{S}^{t} \backslash\left\{q_{ \pm}^{t}\right\}\right)$ to equal $A^{t}$, which we define as the class in $\pi_{2}\left(D^{4}, \tilde{S}^{t} \backslash\left\{q_{ \pm}^{t}\right\}\right)$ that maps to the class of $\left[\left.\varphi \circ u_{0}^{t}\right|_{\partial \mathbb{D}}\right] \in \pi_{1}\left(\tilde{S}^{t} \backslash\left\{q_{ \pm}^{t}\right\}\right)$ under the boundary homomorphism $\partial_{*}$. (This notion of relative homotopy class will be relevant for our discussion of Maslov indices, and our more general set-up in Section 9.) Moreover, we fix three marked points by imposing the condition $u^{t}\left(i^{k}\right) \in \tilde{\ell}_{k}^{t}$, where $\tilde{\ell}_{k}^{t}:=\varphi\left(\ell_{k}^{t}\right), k=0,1,2$, are three leaves of the characteristic foliation $\tilde{S}_{\xi}^{t}=\varphi\left(S_{\xi}^{t}\right)$. Such a holomorphic disc will be called a $t$-level Bishop disc for $\varphi$. The collection

$$
\mathcal{W}_{\varphi}:=\left\{u^{t}: t \in(-1,1), u^{t} \text { is a } t \text {-level Bishop disc for } \varphi\right\}
$$

of all such discs is the moduli space of Bishop discs. We write $\mathcal{M}_{\varphi}(t) \subset \mathcal{W}_{\varphi}$ for the moduli space of $t$-level Bishop discs for $\varphi$.

Section 2 can be read as a description of the structure of $\mathcal{W}_{\text {id }}$. We are now aiming for a similar description of $\mathcal{W}_{\varphi}$ for any contactomorphism $\varphi$ as in Proposition 3.1

In the following propositions we collect some relevant facts about our Bishop discs.

Proposition 4.1. Every Bishop discs for $\varphi$ has Maslov index 2, that is, $\mu\left(A^{t}\right)=2$ for all $t \in(-1,1)$.

Proof. Recall from Section 2.5 that that the Maslov index of $u_{0}^{t}$ equals 2 for all $t \in$ $(-1,1)$. Since the boundary homomorphism $\partial_{*}: \pi_{2}\left(D^{4}, \tilde{S}^{t} \backslash\left\{q_{ \pm}^{t}\right\}\right) \rightarrow \pi_{1}\left(\tilde{S}^{t} \backslash\left\{q_{ \pm}^{t}\right\}\right)$ is an isomorphism, and the Maslov index is invariant under homotopies, it suffices to show that $\partial_{*} A^{t}=\partial_{*}\left[u_{0}^{t}\right]$.

For a given $t$ choose $s \in(-1,1)$ with $1-\delta-t^{2}<s^{2}<1-t^{2}$. Then, since $\varphi=$ id on $\mathcal{U}^{\delta}$, we have $\left[\left.\varphi \circ u_{s}^{t}\right|_{\partial \mathbb{D}}\right]=\left[\left.u_{s}^{t}\right|_{\partial \mathbb{D}}\right]$. Hence, we obtain the following sequence of equalities, where the first one holds by definition of the class $A^{t}$.

$$
\partial_{*} A^{t}=\left[\left.\varphi \circ u_{0}^{t}\right|_{\partial \mathbb{D}}\right]=\left[\left.\varphi \circ u_{s}^{t}\right|_{\partial \mathbb{D}}\right]=\left[\left.u_{s}^{t}\right|_{\partial \mathbb{D}}\right]=\left[\left.u_{0}^{t}\right|_{\partial \mathbb{D}}\right]=\partial_{*}\left[u_{0}^{t}\right] .
$$

This proves the proposition.

In the sequel we have to appeal several times to the maximum principle for holomorphic discs in $\mathbb{C}^{2}$ with boundary on $S^{3}$. We briefly recall the statement in the form needed for our purposes. This maximum principle also explains why we could define our Bishop disc from the outset as maps into the 4-ball $D^{4}$ rather than $\mathbb{C}^{2}$.

Proposition 4.2 (Maximum principle). Let $\Sigma \subset S^{3}$ be a totally real surface in $\mathbb{C}^{2}$, so that the characteristic foliation $\Sigma_{\xi}$ is non-singular. Let $u:(\mathbb{D}, \partial \mathbb{D}) \rightarrow\left(\mathbb{C}^{2}, \Sigma\right)$ be a non-constant holomorphic disc. Then $u$ maps the interior of $\mathbb{D}$ to the interior of the 4-ball $D^{4}$, and $\left.u\right|_{\partial \mathbb{D}}$ is an immersion positively transverse to $\Sigma_{\xi}$.

Proof. A straightforward computation, cf. [10, Lemma 4.11.3], gives

$$
\Delta(\rho \circ u)=2 d \lambda\left(u_{x}, J_{0} u_{x}\right) \geq 0 .
$$

For the definition of $\lambda$ and $\rho$ see Sections 2.1 and 2.2, respectively. Then the strong maximum principle of E. Hopf, cf. [26, Section 2.3], shows that $\rho \circ u=\|u\|^{2}$ cannot attain a maximum in the interior of $\mathbb{D}$. The boundary point lemma (ibid.) implies that $u$ is transverse to $S^{3}$ along the boundary. Since the characteristic foliation $\Sigma_{\xi}$ is given by the intersection of the complex tangencies $\xi$ to $S^{3}$ with $\Sigma$, and $u$ is holomorphic, this forces $\left.u\right|_{\partial \mathbb{D}}$ to be an immersion transverse to $\Sigma_{\xi}$. The statement 
about this immersion being positively transverse to $\Sigma_{\xi}$ follows because the complex structure $J_{0}$ sends the outer normal of $S^{3} \subset \mathbb{C}^{2}$ to a vector positively transverse to $\xi=\operatorname{ker} \alpha$.

Remark. Suppose $M$ is a compact level set $M=\rho^{-1}(1)$ of a smooth function $\rho: W \rightarrow \mathbb{R}$ on an almost complex manifold $(W, J)$, where 1 and nearby values are regular for $\rho$; any compact orientable codimension 1 submanifold of $W$ can be realised in this way. The level set $M$ is called $J$-convex if the complex tangencies to $M$ define a positive contact structure, that is, if $-\left.d \rho \circ J\right|_{T M}$ is a contact form inducing the natural orientation of $M$. One then computes that for a smooth function $f: \mathbb{R} \rightarrow \mathbb{R}$ with $f^{\prime \prime} \gg f^{\prime}>0$ the composition $f \circ \rho$ is strictly plurisubharmonic, i.e. $-d(d(f \circ \rho) \circ J)(X, J X)>0$ for all nonzero tangent vectors $X \in T W$ in a neighbourhood of $M$. From this observation one concludes that the maximum principle holds, mutatis mutandis, for $J$-convex hypersurfaces.

Following Lazzarini [20, cf. [22, Appendix E], we say a holomorphic disc $u$ is simple if there are no two disjoint non-empty open subsets $U, V \subset \mathbb{D}$ such that $u(U)=u(V)$.

Proposition 4.3. All Bishop discs are simple.

Proof. The maximum principle tells us that $\left.u\right|_{\partial \mathbb{D}}: \partial \mathbb{D} \rightarrow \tilde{S}^{t} \backslash\left\{q_{ \pm}^{t}\right\}$ is an immersion transverse to the characteristic foliation $\tilde{S}_{\xi}^{t}$. By the homotopical condition on $\left.u\right|_{\partial \mathbb{D}}$ and the nature of the characteristic foliation $\tilde{S}_{\xi}^{t}$, the restriction of $u$ to $\partial \mathbb{D}$ must then in fact be an embedding.

From this observation one concludes that $u^{t}$ must be simple; since the argument for this last step is of interest elsewhere, we formulate it (in slightly greater generality) as a separate lemma.

Lemma 4.4. Let $(W, J)$ be an almost complex manifold. If $u: \mathbb{D} \rightarrow W$ is a $J$ holomorphic disc such that $\left.u\right|_{\partial \mathbb{D}}$ is an embedding and no interior point of $\mathbb{D}$ is mapped to $u(\partial \mathbb{D})$, then $u$ is simple.

Remark. In the case of our Bishop discs, $\left.u\right|_{\partial \mathbb{D}}$ is an embedding into the strictly pseudoconvex boundary $S^{3}$ of $D^{4}$; the condition on the interior points of $\mathbb{D}$ is then guaranteed by the maximum principle.

Proof of Lemma 4.4. Arguing by contradiction, we assume that there are two disjoint non-empty open subsets $U, V \subset \mathbb{D}$ with $u(U)=u(V)$. By the conditions of the lemma, $U$ and $V$ are disjoint from $\partial \mathbb{D}$. In the proof of [22, Lemma 2.4.1] it is shown that critical points of $u$ in the interior of $\mathbb{D}$ are isolated. Since $\left.u\right|_{\partial \mathbb{D}}$ is an embedding, critical points cannot accumulate near $\partial \mathbb{D}$, so there are only finitely many of them. Choose a non-critical point $z^{*} \in U$ and a half-open line segment $\left[z^{*}, z^{* *}\right)$ from $z^{*}$ to a point $z^{* *} \in \partial \mathbb{D}$, disjoint from the critical points of $u$. Let $P \subset\left[z^{*}, z^{* *}\right)$ be the set of points that have a neighbourhood in $\left[z^{*}, z^{* *}\right)$ consisting of non-injective points for $u$, that is, points $z$ for which there is a different point $z^{\prime} \in \mathbb{D}$ with $u(z)=u\left(z^{\prime}\right)$. By definition $P$ is open in $\left[z^{*}, z^{* *}\right)$, and $z^{*} \in P$, so $P$ is non-empty.

We claim that $P$ is also closed in $\left[z^{*}, z^{* *}\right)$. Indeed, suppose that $\left(z_{\nu}\right)$ is a sequence of points in $P$ converging to some point $z_{0} \in\left[z^{*}, z^{* *}\right)$. Then there are points $w_{\nu} \neq z_{\nu}$ with $u\left(w_{\nu}\right)=u\left(z_{\nu}\right)$. By passing to a subsequence we may assume that the sequence $\left(w_{\nu}\right)$ converges to a point $w_{0} \in \mathbb{D}$. 
Since the point $z_{0}$ is not critical for $u$, the map $u$ is locally injective near $z_{0}$. This implies that $w_{0} \neq z_{0}$, and without loss of generality we may assume that $w_{\nu} \neq z_{0}$ for all $\nu$. Moreover, the assumptions of the lemma imply that $w_{0}$ is an interior point of $\mathbb{D}$.

With this information we are precisely in the situation of [22, Lemma 2.4.3], which tells us that there exists a holomorphic map $\phi$, defined in a neighbourhood of $z_{0}$, such that $\phi\left(z_{0}\right)=w_{0}$ and $u=u \circ \phi$. So $z_{0}$ has a neighbourhood of noninjective points, which means that $z_{0} \in P$, i.e. $P$ is closed in $\left[z^{*}, z^{* *}\right)$.

We conclude that $P=\left[z^{*}, z^{* *}\right)$. In particular, we find a sequence of non-injective points, which we write again as $\left(z_{\nu}\right)$, accumulating at $z^{* *}$. So as before we have points $w_{\nu} \neq z_{\nu}$ with $u\left(w_{\nu}\right)=u\left(z_{\nu}\right)$, with $\left(w_{\nu}\right)$ converging to some point $w_{0}$. Then $u\left(w_{0}\right)=u\left(z^{* *}\right)$, which implies $w_{0}=z^{* *}$. But $u$ is locally injective near $z^{* *}$, so it is impossible for the two sequences $\left(z_{\nu}\right)$ and $\left(w_{\nu}\right)$ of corresponding non-injective points to accumulate at $z^{* *}$. This contradiction proves the lemma.

Remark. Instead of asking for $\left.u\right|_{\partial \mathbb{D}}$ to be an embedding, it suffices to require that $\left.u\right|_{\partial \mathbb{D}}$ be injective. Then the set of critical points is still finite, see [20, Theorem 3.5], and the proof above goes through verbatim.

Proposition 4.5. All Bishop discs are embedded and mutually disjoint.

Proof. Since all Bishop discs are simple, the results from Section 9 below on the positivity of intersections apply. In that section we define a so-called embedding defect $D$ for holomorphic discs. This embedding defect depends only on the relative homotopy class $A^{t}=\left[u^{t}\right] \in \pi_{2}\left(D^{4}, \tilde{S}^{t} \backslash\left\{q_{ \pm}^{t}\right\}\right)$, and it equals zero if and only if $u^{t}$ is embedded (Theorem 9.4).

For each $t \in(-1,1)$, the discs $u_{s}^{t}$ with $s$ in the range given by $1-\delta-t^{2}<$ $s^{2}<1-t^{2}$ belong to our moduli space $\mathcal{M}_{\varphi}(t)$ of $t$-level Bishop discs. In particular, $\mathcal{M}_{\varphi}(t)$ contains at least two disjoint embedded holomorphic discs. It follows that $D\left(A^{t}\right)=0$ and that all Bishop discs are embedded.

Given two Bishop discs of distinct levels, their boundary curves are disjoint. Then the intersection number of the two discs equals the linking number of their boundary curves in $S^{3}$, which is zero. By positivity of intersections (at interior points), see [23, Theorem 7.1], it follows that the two discs must be disjoint.

It remains to show that the same holds true for Bishop discs at one and the same level $t$. From the defining equation of the embedding defect in Section 9, and with $\mu\left(A^{t}\right)=2$, it follows that the self-intersection number $A^{t} \bullet A^{t}$, as defined in Section 8, equals zero for every $t$. (Alternatively, this is a consequence of the existence of two disjoint $t$-level Bishop discs.) With positivity of intersections (Theorem 9.2) we infer that any two $t$-level Bishop discs are either disjoint, or their images coincide. Since we prescribed the images of three marked points on each Bishop disc, two Bishop discs with the same image are actually identical.

Corollary 4.6. If the boundary of a t-level Bishop disc $u$ hits the set $\overline{\mathcal{U}}^{\delta}$, then $u=u_{s}^{t}$ for some $s$ with $1-\delta-t^{2} \leq s^{2}<1-t^{2}$.

Proof. The contactomorphism $\varphi$ is the identity on $\mathcal{U}$, which contains $\overline{\mathcal{U}}^{\delta}$. That latter set is foliated by the boundary circles of standard discs $u_{s}^{t}$. Now invoke the preceding proposition. 
It is therefore opportune to restrict attention to the truncated moduli space

$$
\begin{aligned}
\mathcal{W}_{\varphi}^{\delta} & :=\left\{u^{t}: t \in[-\sqrt{1-\delta}, \sqrt{1-\delta}]\right. \\
& \left.u^{t} \text { is a } t \text {-level Bishop disc for } \varphi \text { such that } u^{t}(\partial \mathbb{D}) \subset S^{3} \backslash \mathcal{U}^{\delta}\right\} .
\end{aligned}
$$

In Sections 6 and 7 we shall prove that this moduli space is a compact manifold with boundary. Working with this truncated moduli space allows us to circumvent all subtleties along the singular set $K$.

The following uniform energy estimate will be one ingredient in that compactness argument.

Proposition 4.7. The symplectic energy of all Bishop discs for $\varphi$ is uniformly bounded by a constant depending only on $\varphi$.

Proof. By Proposition 4.5 any Bishop disc $u$ is an embedding. So with Stokes's theorem and the transformation formula, the energy $E(u)$ can be computed as

$$
E(u)=\int_{\mathbb{D}} u^{*} \omega=\int_{u(\partial \mathbb{D})} \alpha=\int_{\varphi^{-1} \circ u(\partial \mathbb{D})} \varphi^{*} \alpha .
$$

The contactomorphism $\varphi$ pulls back the contact form $\alpha$ to $f \alpha$, where $f: S^{3} \rightarrow$ $\mathbb{R}^{+}$is a smooth function bounded above by some constant $C=C(\varphi)$. By the maximum principle, $\left.u\right|_{\partial \mathbb{D}}$ is positively transverse to $\xi=\operatorname{ker} \alpha$, and the same holds for $\left.\varphi^{-1} \circ u\right|_{\partial \mathbb{D}}$. It follows that

$$
E(u) \leq C \int_{\varphi^{-1} \circ u(\partial \mathbb{D})} \alpha .
$$

The 2-sphere $S^{t}$, which contains the embedded circle $\varphi^{-1} \circ u(\partial \mathbb{D})$, is naturally oriented by the area form

$$
\sigma^{t}:=\frac{1}{\sqrt{1-t^{2}}}\left(x_{1} d y_{1} \wedge d x_{2}+y_{1} d x_{2} \wedge d x_{1}+x_{2} d x_{1} \wedge d y_{1}\right)
$$

the total area of $S^{t}$ equals $4 \pi\left(1-t^{2}\right)$. Let $D^{t}$ be the 2 -disc in $S^{t}$ (with the induced orientation) whose oriented boundary equals $\varphi^{-1} \circ u(\partial \mathbb{D})$. Then

$$
E(u) \leq C \int_{\partial D^{t}} \alpha=C \int_{D^{t}} \omega .
$$

On $T S^{t}$ we have $x_{1} d x_{1}+y_{1} d y_{1}+x_{2} d x_{2}=0$. Using this, one finds that

$$
x_{2} \sigma^{t}=\sqrt{1-t^{2}} d x_{1} \wedge d y_{1}=\sqrt{1-t^{2}} \omega \text { on } T S^{t} .
$$

Hence, with $x_{2} \leq \sqrt{1-t^{2}}$ we conclude

$$
E(u) \leq \frac{C}{\sqrt{1-t^{2}}} \int_{D^{t}} x_{2} \sigma^{t} \leq C \int_{S^{t}} \sigma^{t}=4 \pi C\left(1-t^{2}\right) \leq 4 \pi C .
$$

This is the desired uniform estimate.

Remark. The symplectic energy $E(u)$ of a holomorphic disc equals its Dirichlet energy $(1 / 2) \int_{\mathbb{D}}|\nabla u|^{2}$ see [22, Section 2.2]. Since the symplectic energy is, by its definition, invariant under reparametrisations, it follows that the Dirichlet energy is invariant under conformal reparametrisations. 


\section{From the FILLing TO THE EXTENSION}

By taking the results on compactness and transversality from Sections 6 and 7 for granted, we can now determine the truncated moduli space.

Recall from Section 2.7 that $Q^{\delta}$ is a closed 2-disc in $S^{3}$ defined by $Q^{\delta}=$ $\left\{u_{s}^{t}(1):(s, t) \in \mathbb{D}_{1-\delta}\right\}$, where $\mathbb{D}_{1-\delta}$ denotes the closed disc $\left\{s^{2}+t^{2} \leq 1-\delta\right\}$. Hence, if $u$ is a Bishop disc for $\varphi$, our condition on the marked points implies $u(1) \in \varphi\left(Q^{\delta}\right)$.

Proposition 5.1. The evaluation map

$$
\begin{aligned}
\mathrm{ev}_{1}: \quad \mathcal{W}_{\varphi}^{\delta} & \longrightarrow \varphi\left(Q^{\delta}\right) \\
u & \longmapsto u(1)
\end{aligned}
$$

is a diffeomorphism.

This proposition will be proved at the end of Section 7 once we have established the relevant compactness and transversality results.

We now want to define a notion of holomorphic filling of $S^{3}$ (with singular set $K$ ) with respect to a contactomorphism $\varphi$ of $\left(S^{3}, \xi\right)$ that subsumes, for the identity map, the standard filling $F_{\text {st }}$. In the sequel, the contactomorphism $\varphi$ is always taken as a given and will be omitted from the notation.

Definition. A holomorphic filling of $S^{3}$ is a diffeomorphism

$$
F:(\mathbb{D} \times \operatorname{Int} \mathbb{D}, \partial \mathbb{D} \times \operatorname{Int} \mathbb{D}) \longrightarrow\left(D^{4} \backslash K, S^{3} \backslash K\right)
$$

with the following properties:

(1) $\bar{\partial} F(., s, t)=0$ for all $(s, t) \in \operatorname{Int} \mathbb{D}$;

(2) $F$ extends continuously to a map (still denoted $F$ ) defined on $\mathbb{D} \times \mathbb{D}$ such that $F(\mathbb{D} \times \partial \mathbb{D})=K$;

(3) for all $(z, s, t) \in \partial \mathbb{D} \times \operatorname{Int} \mathbb{D}$ we have $F(z, s, t) \in \tilde{S}^{t} \backslash\left\{q_{ \pm}^{t}\right\}$, and as $s$ tends to $\pm \sqrt{1-t^{2}}$, the limit of $F(z, s, t)$ equals $q_{ \pm}^{t}$ for each $z \in \partial \mathbb{D}$.

Observe that for $t \in(-1,1)$, the punctured 2-sphere $\tilde{S}^{t} \backslash\left\{q_{ \pm}^{t}\right\}$ will be foliated by the boundary circles $F(\partial \mathbb{D}, s, t)$ of the holomorphic $\operatorname{discs} F(\mathbb{D}, s, t)$, where $|s|<$ $\sqrt{1-t^{2}}$.

We now want to construct such a holomorphic filling with the help of the moduli space $\mathcal{W}_{\varphi}^{\delta}$. The map described in the next proposition is defined thanks to Proposition 5.1

Proposition 5.2. The map

$$
F^{\delta}:\left(\mathbb{D} \times \mathbb{D}_{1-\delta}, \partial \mathbb{D} \times \mathbb{D}_{1-\delta}\right) \longrightarrow\left(D^{4}, S^{3}\right)
$$

defined by

is an embedding.

$$
(z, s, t) \longmapsto\left(\mathrm{ev}_{1}^{-1} \circ \varphi \circ u_{s}^{t}(1)\right)(z)
$$

Again, the proof of this proposition relies on compactness and transversality statements and will be given at the end of Section 7 .

For $(s, t)$ in a neighbourhood of $\partial \mathbb{D}_{1-\delta} \subset \mathbb{D}_{1-\delta}$ we have $F^{\delta}(z, s, t)=u_{s}^{t}(z)=$ $F_{\text {st }}(z, s, t)$. Therefore we obtain a holomorphic filling by setting

$$
F=\left\{\begin{array}{lll}
F^{\delta} & \text { on } & \mathbb{D} \times \mathbb{D}_{1-\delta}, \\
F_{\text {st }} & \text { on } & \mathbb{D} \times\left\{1-\delta \leq s^{2}+t^{2} \leq 1\right\} .
\end{array}\right.
$$


By construction, the maps $F_{\text {st }}$ and $F$ restrict to diffeomorphisms

$$
f, f_{\mathrm{st}}:\left(S^{1} \times \mathbb{D}_{1-\delta}, S^{1} \times \partial \mathbb{D}_{1-\delta}\right) \longrightarrow\left(S^{3} \backslash \mathcal{U}^{\delta}, \partial \mathcal{U}^{\delta}\right) .
$$

Observe that

$$
f \circ f_{\mathrm{st}}^{-1}: S^{3} \backslash \mathcal{U}^{\delta} \longrightarrow S^{3} \backslash \mathcal{U}^{\delta}
$$

is a diffeomorphism equal to the identity near the boundary of $S^{3} \backslash \mathcal{U}^{\delta}$. By slight abuse of notation, we regard $f \circ f_{\text {st }}^{-1}$ as a diffeomorphism of $S^{3}$, equal to the identity in a neighbourhood of $\overline{\mathcal{U}}^{\delta}$.

Lemma 5.3. The two diffeomorphisms $f \circ f_{\mathrm{st}}^{-1}$ and $\varphi$ of $S^{3}$ are isotopic relative to a neighbourhood of $\overline{\mathcal{U}}^{\delta}$.

Before proving this lemma, we show that Cerf's theorem is now an immediate consequence.

Proof of Cerf's theorem $\Gamma_{4}=0$. Let $\mathcal{V}^{\delta}$ be the open subset of $D^{4}$ defined by

$$
\mathcal{V}^{\delta}:=F_{\mathrm{st}}\left(\mathbb{D} \times\left\{1-\delta<s^{2}+t^{2} \leq 1\right\}\right) .
$$

Notice that $\mathcal{V}^{\delta} \cap S^{3}=\mathcal{U}^{\delta}$. Define $G: D^{4} \rightarrow D^{4}$ by

$$
G:=\left\{\begin{array}{lll}
F \circ F_{\mathrm{st}}^{-1} & \text { on } & D^{4} \backslash \mathcal{V}^{\delta} \\
\text { id } & \text { on } & \mathcal{V}^{\delta}
\end{array}\right.
$$

This is a diffeomorphism of $D^{4}$ that restricts to $f \circ f_{\mathrm{st}}^{-1}$ on $S^{3}$.

Now an extension of the diffeomorphism $\varphi: S^{3} \rightarrow S^{3}$ to $D^{4}$ is defined by sweeping out the isotopy to $f \circ f_{\mathrm{st}}^{-1}$ over a collar neighbourhood of $S^{3}$ in $D^{4}$, and then extending over the remaining 4-ball as the diffeomorphism $G$.

Proof of Lemma 5.3. Consider the diffeomorphism $\chi$ of $S^{1} \times \mathbb{D}_{1-\delta}$ defined as the composition $\chi=f_{\text {st }}^{-1} \circ \varphi^{-1} \circ f$. For ease of notation we identify $S^{1}$ with $\mathbb{R} / 2 \pi \mathbb{Z}$ and write $\chi=\chi(\theta, s, t)$. This diffeomorphism $\chi$ equals the identity near the boundary. Our condition on the marked point $1 \in S^{1}=\partial \mathbb{D}$ (corresponding to $\theta=0$ ) translates into saying that $\chi(0, s, t)=(0, s, t)$ for all $(s, t) \in \mathbb{D}_{1-\delta}$.

Write the components of $\chi$ as $\chi=\left(\chi^{1}, \chi^{2}, \chi^{3}\right)$. The $t$-level Bishop discs for $\varphi$ have boundary in $\tilde{S}^{t}=\varphi\left(S^{t}\right)$. This implies $\chi^{3}(\theta, s, t)=t$.

The map $f_{\text {st }}$ sends the $s$-curves to the leaves of the characteristic foliation $S_{\xi}^{t}$, hence $\varphi \circ f_{\text {st }}$ sends those curves to the leaves of $\tilde{S}_{\xi}^{t}$. On the other hand, the $\theta$-curves are mapped by $f$ to curves positively transverse to the leaves of $\tilde{S}_{\xi}^{t}$, thanks to the maximum principle. We conclude $\partial_{\theta} \chi^{1}>0$. This implies that, for each fixed $t$, the images of the $\theta$-curves under $\chi$ are graphs of functions $s=s(\theta)$.

The idea mentioned in Section 2 of isotoping the boundaries of the Bishop discs for $\varphi$ to the images under $\varphi$ of the boundaries of the standard Bishop discs translates into the isotopy

$$
(\theta, s, t) \longmapsto\left(\chi^{1},(1-\sigma) \chi^{2}+\sigma s, t\right), \sigma \in[0,1] .
$$

The condition $\partial_{\theta} \chi^{1}>0$ and the resulting fact that $\chi$ maps each $\theta$-curve to a graph guarantee that this is indeed an isotopy, stationary near the boundary; thanks to this boundary behaviour it is enough to observe that for each $\sigma \in[0,1]$ the given map constitutes an injective immersion. 
For each fixed $s, t$ we may regard the function $\theta \mapsto \chi^{1}(\theta, s, t)$ as a strictly increasing function $[0,2 \pi] \rightarrow[0,2 \pi]$ sending both 0 and $2 \pi$ to itself. With this interpretation, an isotopy from the map above for $\sigma=1$ to the identity is given by

$$
(\theta, s, t) \longmapsto\left((1-\tau) \chi^{1}+\tau \theta, s, t\right), \quad \tau \in[0,1] .
$$

Again, this isotopy is stationary near the boundary.

In conclusion, we have found an isotopy from $\chi$ to the identity, stationary near the boundary, which is equivalent to having the isotopy claimed in the lemma.

\section{Compactness}

In the present section we wish to show that the truncated moduli space $\mathcal{W}_{\varphi}^{\delta}$ is compact; in the next section we find that it is a manifold with boundary by proving surjectivity of the relevant linearised Cauchy-Riemann operator. For both these analytical questions it is convenient and customary to work not with smooth maps, but with the space $W^{1, p}$, for some $p>2$, of (equivalence classes of) $L^{p}$-functions whose first partial derivatives in the weak sense exist and are $p$-integrable.

This approach is justified by elliptic regularity, see [22, Appendix B]: (i) a holomorphic curve of class $W^{1, p}$ will actually be smooth up to the boundary; (ii) a sequence of holomorphic curves that converges in the $W^{1, p}$-norm also converges locally uniformly with all its derivatives.

Thus, we now equip the moduli space $\mathcal{W}_{\varphi}$ with the topology induced by the $W^{1, p}$-norm, $p>2$, on maps $\mathbb{D} \rightarrow D^{4}$. In the Banach space $W^{1, p}\left(\mathbb{D}, \mathbb{R}^{4}\right)$ we then have tools such as the implicit function theorem at our disposal.

Let $\left(u_{\nu}\right)$, with $u_{\nu}$ of level $t_{\nu}$, be a sequence in the truncated moduli space $\mathcal{W}_{\varphi}^{\delta}$ converging to $u_{0} \in W^{1, p}\left(\mathbb{D}, D^{4}\right)$. By passing to a subsequence we may assume that the sequence of levels $\left(t_{\nu}\right)$ converges to some $t_{0} \in[-\sqrt{1-\delta}, \sqrt{1-\delta}]$. By what we just said, $\left(u_{\nu}\right)$ converges with all derivatives to $u_{0}$. Thus, $u_{0}$ is again a holomorphic disc. Moreover, the boundary circles $u_{\nu}(\partial \mathbb{D}) \subset \tilde{S}^{t_{\nu}} \backslash\left\{q_{ \pm}^{t_{\nu}}\right\}$ are homotopically nontrivial and stay outside the neighbourhood $\mathcal{U}^{\delta}$ of the poles $q_{ \pm}^{t}$. So the same will be true for $u_{0}$. In other words, $\mathcal{W}_{\varphi}^{\delta}$ is a closed subset of $W^{1, p}\left(\mathbb{D}, D^{4}\right)$.

Our proof of the following proposition uses methods from [14, cf. in particular the proof of Proposition 3.15 in that paper.

Proposition 6.1. The truncated moduli space $\mathcal{W}_{\varphi}^{\delta}$ is compact.

Proof. Let $\left(u_{\nu}\right)$ be a sequence in $\mathcal{W}_{\varphi}^{\delta}$, where $u_{\nu}$ is of level $t_{\nu}$. After passing to a subsequence we may assume that $t_{\nu} \rightarrow t_{0} \in[-\sqrt{1-\delta}, \sqrt{1-\delta}]$. By [22, Theorem B.4.2], in order to prove compactness (i.e. to find a converging subsequence with respect to the $W^{1, p}$-norm), we need to establish a uniform $L^{p}$-bound for the sequence $\left(\left|\nabla u_{\nu}\right|\right)$. We claim that the sequence $\left(\left|\nabla u_{\nu}\right|\right)$ is uniformly bounded even in the supremum norm on the closed disc $\mathbb{D}$.

Arguing by contradiction, assume that such a uniform bound does not exist. We can then find a sequence of points $z_{\nu} \rightarrow z_{0}$ in $\mathbb{D}$ such that $\left|\nabla u_{\nu}\left(z_{\nu}\right)\right| \rightarrow \infty$. The classical convergence theorems of Montel and Weierstraß preclude this at interior points: the maximum principle provides us with a $C^{0}$-bound on $\left(u_{\nu}\right)$ needed for Montel's theorem, which then guarantees the existence of a locally uniformly convergent subsequence on Int $\mathbb{D}$; the theorem of Weierstraß tells us that the limit function $u$ is holomorphic and $\nabla u_{\nu}\left(z_{\nu}\right) \rightarrow \nabla u\left(z_{0}\right)$, which means that this sequence of gradients remains bounded. 
So necessarily $z_{0} \in \partial \mathbb{D}$. Choose a conformal map from the closed upper halfplane $\mathbb{H} \subset \mathbb{C}$ to $\mathbb{D} \backslash\left\{-z_{0}\right\}$ that sends 0 to $z_{0}$. (This extends to a conformal map from $\mathbb{H} \cup\{\infty\} \subset \hat{\mathbb{C}}:=\mathbb{C} \cup\{\infty\}$ to $\mathbb{D}$.) The differential of this map is bounded from above and below near $0 \in \mathbb{H}$, so by precomposing with this conformal map we may regard the $u_{\nu}$, by slight abuse of notation, as maps

$$
u_{\nu}:(\mathbb{H}, \mathbb{R}) \longrightarrow\left(D^{4}, \tilde{S}^{t_{\nu}} \backslash\left\{q_{ \pm}^{t_{\nu}}\right\}\right),
$$

and the sequence $\left(z_{\nu}\right)$ as a sequence in $\mathbb{H}$ converging to 0 , still satisfying

$$
R_{\nu}:=\left|\nabla u_{\nu}\left(z_{\nu}\right)\right| \rightarrow \infty .
$$

Notice that by Proposition 4.7 and the remark following it we have a uniform bound on the Dirichlet energy, i.e. there is a constant $C$ such that

$$
\frac{1}{2} \int_{\mathbb{H}}\left|\nabla u_{\nu}\right|^{2} \leq C \text { for all } \nu \in \mathbb{N} .
$$

Choose a sequence $\varepsilon_{\nu} \searrow 0$ such that $\varepsilon_{\nu} R_{\nu} \rightarrow \infty$, e.g. $\varepsilon_{\nu}=1 / \ln R_{\nu}$. Hofer's Lemma [16, Lemma 6.4.5], applied to the continuous function $z \mapsto\left|\nabla u_{\nu}(z)\right|$, allows us to modify the sequences $\left(z_{\nu}\right)$ and $\left(\varepsilon_{\nu}\right)$ in such a way that, in addition to the previous conditions, we have the uniform estimates

$$
\left|\nabla u_{\nu}(z)\right| \leq 2 R_{\nu} \text { for all } z \in \mathbb{H} \text { with }\left|z-z_{\nu}\right| \leq \varepsilon_{\nu} .
$$

After passing to a further subsequence, we may assume that

$$
R_{\nu} \operatorname{dist}\left(z_{\nu}, \partial \mathbb{H}\right) \rightarrow r \text { for some } r \in[0, \infty] .
$$

When we write $z_{\nu}=x_{\nu}+i y_{\nu}$, this reads as $R_{\nu} y_{\nu} \rightarrow r$. We shall deal separately with two cases: either $r<\infty$ or $r=\infty$.

First case: $r<\infty$. (i) In a first step we are going to show that a rescaled subsequence of $\left(u_{\nu}\right)$ converges to a holomorphic disc. In a second step we then prove that this limit disc has contradictory properties. Replace the sequence $\left(u_{\nu}\right)$ by the rescaled sequence $\left(w_{\nu}\right)$, defined by

$$
w_{\nu}(z):=u_{\nu}\left(x_{\nu}+z / R_{\nu}\right) .
$$

Since this amounts to a conformal change of parameters, the Dirichlet energy of the $w_{\nu}$ is still bounded by $C$. The uniform gradient estimate now becomes, with $\zeta_{\nu}:=i R_{\nu} y_{\nu}$,

$$
\left|\nabla w_{\nu}(z)\right| \leq 2 \text { for all } z \in \mathbb{H} \text { with }\left|z-\zeta_{\nu}\right| \leq \varepsilon_{\nu} R_{\nu} .
$$

Notice that $\zeta_{\nu} \rightarrow$ ir and $\left|\nabla w_{\nu}\left(\zeta_{\nu}\right)\right|=1$.

We wish to apply [22, Theorem B.4.2] in order to extract a $C_{\text {loc }}^{\infty}$-converging subsequence of $\left(w_{\nu}\right)$. That theorem allows us to have varying almost complex structures, but requires a fixed boundary condition. Therefore we modify our setup as follows. Choose a sequence $\left(\Psi_{\nu}\right)$ of diffeomorphisms of $\mathbb{C}^{2}$ with the following properties:

(1) $\Psi_{\nu}$ equals the identity outside a ball of radius 2 ,

(2) $\Psi_{\nu} \rightarrow$ id in the $C^{\infty}$-topology,

(3) $\Psi_{\nu}\left(\tilde{S}^{t_{\nu}}\right)=\tilde{S}^{t_{0}}$ and $\Psi_{\nu}\left(q_{ \pm}^{t_{\nu}}\right)=q_{ \pm}^{t_{0}}$.

Such $\Psi_{\nu}$ can be constructed by suitably cutting off the gradient flow of the function $H \circ \varphi^{-1}$ on $S^{3}$, where $H$ is the height function defined in Section 2. 
Set $\hat{w}_{\nu}:=\Psi_{\nu} \circ w_{\nu}$ and $J_{\nu}:=T \Psi_{\nu} \circ J_{0} \circ T \Psi_{\nu}^{-1}$, so that $J_{\nu} \rightarrow J_{0}$. Thus, we now have a sequence $\left(\hat{w}_{\nu}\right)$ of $J_{\nu}$-holomorphic maps

$$
\hat{w}_{\nu}:(\mathbb{H}, \mathbb{R}) \longrightarrow\left(D^{4}, \tilde{S}^{t_{0}} \backslash\left\{q_{ \pm}^{t_{0}}\right\}\right) .
$$

The $\hat{w}_{\nu}$ are still subject to the same estimates as the $w_{\nu}$, possibly after replacing the relevant constants by larger ones depending only on the sequence $\left(\Psi_{\nu}\right)$.

Now [22, Theorem B.4.2] does indeed permit us to select a subsequence of $\left(\hat{w}_{\nu}\right)$ converging in $C_{\mathrm{loc}}^{\infty}$ to a non-constant $J_{0}$-holomorphic map

$$
w:(\mathbb{H}, \mathbb{R}) \longrightarrow\left(D^{4}, \tilde{S}^{t_{0}} \backslash\left\{q_{ \pm}^{t_{0}}\right\}\right) .
$$

The cited theorem requires the boundary condition to be given by a closed totally real submanifold. No problems arise from our working with the punctured 2-sphere $\tilde{S}^{t_{0}} \backslash\left\{q_{ \pm}^{t_{0}}\right\}$, since we know a priori that $w_{\nu}$ sends the boundary circle $\partial \mathbb{H} \cup\{\infty\}$ to $\tilde{S}^{t_{\nu}} \backslash \mathcal{U}^{\delta}$

The $C_{\mathrm{loc}}^{\infty}$-convergence ensures that the energy of the limit $w$ is estimated from above by $C$. By removal of singularities, see [20, Appendix B] and [27, Theorem 4.7.3], $w$ extends to a holomorphic map defined on $\mathbb{H} \cup\{\infty\}$. By reversing our change of conformal parameters, we regard this again as a map

$$
w:(\mathbb{D}, \partial \mathbb{D}) \longrightarrow\left(D^{4}, \tilde{S}^{t_{0}} \backslash\left\{q_{ \pm}^{t_{0}}\right\}\right),
$$

i.e. we have an honest holomorphic disc.

(ii) We now want to show that such a holomorphic disc $w$ cannot possibly exist. As we saw in the proof of Proposition 4.3, the map $\left.u\right|_{\partial \mathbb{D}}$ is an embedding transverse to the characteristic foliation $\tilde{S}_{\xi}^{t_{0}}$.

The leaf space of the characteristic foliation $\tilde{S}_{\xi}^{t}$, for each $t \in(-1,1)$ and with the singular points $q_{ \pm}^{t}$ removed, can be identified with $S^{1}$, where $\theta \in S^{1}$ corresponds to the leaf $s \mapsto \varphi \circ F_{\mathrm{st}}\left(e^{i \theta}, s, t\right)$. Let $\sigma$ be the homogeneous measure on $S^{1}$ of total measure 1 , say.

We now regard $\left.u\right|_{\partial \mathbb{D}}$ as a map from $\partial \mathbb{D}$ to the leaf space $S^{1}$ of $\tilde{S}_{\xi}^{t_{0}}$; the maps $\left.u_{\nu}\right|_{\partial \mathbb{H}}$ and $\left.w_{\nu}\right|_{\partial \mathbb{H}}$ will be viewed similarly. Choose a segment $I \subset S^{1} \backslash\left\{-z_{0}\right\}$ containing $z_{0}$ such that $\sigma(u(I)) \geq 15 / 16$. Under the identification of $\partial \mathbb{D} \backslash\left\{-z_{0}\right\}$ with $\partial \mathbb{H}$ we take $I$ of the form $I=[-R, R]$.

Because of the $C_{\mathrm{loc}}^{\infty}$-convergence we have $\sigma\left(w_{\nu}(I)\right) \geq 7 / 8$ for $\nu$ sufficiently large. With $I_{\nu}:=\left[x_{\nu}-R / R_{\nu}, x_{\nu}+R / R_{\nu}\right]$ this means that $\sigma\left(u_{\nu}\left(I_{\nu}\right)\right) \geq 7 / 8$. The length of the interval $I_{\nu}$ tends to zero, so at least one of the three intervals in $\partial \mathbb{H} \cup\{\infty\}=\partial \mathbb{D}$ between the three marked points $1, i,-1$ will be disjoint from $I_{\nu}$ for $\nu$ sufficiently large; call this interval $I^{\prime}$. The condition on marked points of our holomorphic discs implies that $\sigma\left(u_{\nu}\left(I^{\prime}\right)\right)=\sigma\left(I^{\prime}\right)$, which equals $1 / 4$ or $1 / 2$. Hence $\sigma\left(u_{\nu}(I)\right)+$ $\sigma\left(u_{\nu}\left(I^{\prime}\right)\right)>1$, contradicting the fact that $u_{\nu}(I) \cap u_{\nu}\left(I^{\prime}\right)=\emptyset$.

This contradiction completes the proof in the first case.

Second case: $r=\infty$. In this case we define the rescaled sequence $\left(w_{\nu}\right)$ by

$$
w_{\nu}(z):=u_{\nu}\left(z_{\nu}+z / R_{\nu}\right) \text { for } z=x+i y \text { with } y \geq-y_{\nu} R_{\nu}
$$

Then $\left|\nabla w_{\nu}(0)\right|=1$, the Dirichlet energy of the $w_{\nu}$ is bounded by $C$, and we have the uniform estimate

$$
\left|\nabla w_{\nu}(z)\right| \leq 2 \text { for all } z \in \mathbb{H} \text { with }\left|z-z_{\nu}\right| \leq \varepsilon_{\nu} R_{\nu} .
$$


Again we may appeal to [22, Theorem B.4.2]; this now gives us a subsequence of $\left(w_{\nu}\right)$ that converges in $C_{\text {loc }}^{\infty}$ to a holomorphic map $w: \mathbb{C} \rightarrow \mathbb{C}^{2}$, which is bounded by 1 and non-constant because of $|\nabla w(0)|=1$. By the classical Hebbarkeitssatz of Riemann, now applied to a conformal chart of $\hat{\mathbb{C}}=\mathbb{C} \cup\{\infty\}$ near $\infty$, we obtain a non-constant holomorphic sphere $w: S^{2} \rightarrow \mathbb{C}^{2}$, in contradiction to the maximum principle.

Remark. With a view towards potential generalisations of this result it is opportune to remark that the references to classical theorems of complex analysis can be substituted by results that hold for almost complex structures tamed by a symplectic form.

For proving that $\left(\left|\nabla u_{\nu}\right|\right)$ is uniformly bounded one may replace the reference to the convergence theorems of Montel and Weierstraß by arguments from 22, Section 4.2]. For $z_{\nu} \rightarrow z_{0} \in \operatorname{Int} \mathbb{D}$ and $\left|\nabla u_{\nu}\left(z_{\nu}\right)\right| \rightarrow \infty$ these arguments would allow one to infer the existence of a bubble, i.e. a non-constant holomorphic sphere in $\mathbb{C}^{2}$, in violation of the maximum principle.

In place of Riemann's Hebbarkeitssatz one may cite [22, Theorem 4.1.2].

\section{TRANSVERSALity}

Here is the main result of the present section:

Proposition 7.1. The truncated moduli space of Bishop discs $\mathcal{W}_{\varphi}^{\delta}$ is a 2-dimensional manifold with boundary $\partial \mathcal{W}_{\varphi}^{\delta}=\left\{u_{s}^{t}: s^{2}+t^{2}=1-\delta\right\}$.

For the proof of this proposition we shall establish automatic transversality results as in [11] and [15]; the attribute 'automatic' refers to the fact that no perturbation of the (almost) complex structure is required to guarantee surjectivity of the Fredholm operator in question.

We initially drop the condition on the three marked points. Thus, write $\widetilde{\mathcal{W}}_{\varphi}$ for the free moduli space of all $t$-level Bishop discs for $\varphi$, where $t$ varies in the interval $(-1,1)$, but now without any restriction on the image of the points $1, i,-1$. Our aim will be to show that $\widetilde{\mathcal{W}}_{\varphi}$ is a 5 -dimensional manifold. Once this has been achieved, the following simple lemma allows us to deduce that our original moduli space $\mathcal{W}_{\varphi}$ is a 2 -dimensional manifold.

The tangent space $T_{u} W^{1, p}\left(\mathbb{D}, \mathbb{R}^{4}\right)$ to the Banach space $W^{1, p}\left(\mathbb{D}, \mathbb{R}^{4}\right)$ at some point $u$ of that Banach space can of course be naturally identified with the Banach space itself. When the target space is not a linear space, but some manifold $W$, the tangent space $T_{u} W^{1, p}(\mathbb{D}, W)$ to the Banach manifold $W^{1, p}(\mathbb{D}, W)$ is the space of $W^{1, p}$-sections of the pullback bundle $u^{*} T W$, cf. [22, Chapter 3] or [2, Section 6.2]; in other words, a tangent vector $\eta$ at $u$ is a map $\eta: \mathbb{D} \rightarrow T \mathbb{R}^{4}$ with $\eta(z) \in T_{u(z)} \mathbb{R}^{4}$. We also want to consider Banach manifolds of relative maps $(\mathbb{D}, \partial \mathbb{D}) \rightarrow\left(\mathbb{R}^{4}, \Sigma\right)$, where $\Sigma \subset S^{3}$ is a totally real submanifold. Then tangent vectors are sections of the bundle pair (cf. Section 2) $u^{*}\left(T \mathbb{R}^{4}, T \Sigma\right.$ ). The details of this relative case are worked out in [32, Section 3.1]. The key point here is to choose an auxiliary metric for which $\Sigma$ is totally geodesic in $\mathbb{R}^{4}$. Then the composition of a section of $u^{*}\left(T \mathbb{R}^{4}, T \Sigma\right)$ with the exponential map for this metric will be a map $(\mathbb{D}, \partial \mathbb{D}) \rightarrow\left(\mathbb{R}^{4}, \Sigma\right)$.

In particular, if $m_{\tau}, \tau \in(-\varepsilon, \varepsilon)$, is a holomorphic reparametrisation of $\mathbb{D}$, with $m_{0}=\mathrm{id}_{\mathbb{D}}$, we may regard $\eta:=\left.(d / d \tau)\right|_{\tau=0}\left(u \circ m_{\tau}\right)$ as a tangent vector in this sense; here $\eta(z)$ is actually tangent to $u(\mathbb{D})$ at $u(z)$; and for $z \in \partial \mathbb{D}$, tangent to $u(\partial \mathbb{D})$.

See Section 2.7 for the definition of $Q_{k}$ used in the following lemma. 
Lemma 7.2. The evaluation map

$$
\begin{array}{cccc}
\mathrm{ev}_{1, i,-1}: \quad \widetilde{\mathcal{W}}_{\varphi} & \longrightarrow & S^{3} \times S^{3} \times S^{3} \\
u & \longmapsto(u(1), u(i), u(-1))
\end{array}
$$

is transverse to $\tilde{Q}_{0} \times \tilde{Q}_{1} \times \tilde{Q}_{2}$, where $\tilde{Q}_{k}:=\varphi\left(Q_{k}\right)$.

Proof. Observe that $\mathcal{W}_{\varphi}=\operatorname{ev}_{1, i,-1}^{-1}\left(\tilde{Q}_{0} \times \tilde{Q}_{1} \times \tilde{Q}_{2}\right)$, so we need to investigate the differential of the evaluation map at points $u \in \mathcal{M}_{\varphi}(t) \subset \mathcal{W}_{\varphi}$. This differential sends a tangent vector $\eta$ to the tangent vector $(\eta(1), \eta(i), \eta(-1))$ at the point $(u(1), u(i), u(-1)) \in S^{3} \times S^{3} \times S^{3}$.

Let $m_{\tau}^{k}, k=0,1,2, \tau \in(-\varepsilon, \varepsilon)$, be the 1-parameter family of Möbius transformations of $\mathbb{D}$ uniquely determined by the condition that the marked point $i^{k}$ be sent to $e^{i \tau} i^{k}$ and the other two marked points be fixed.

Recall that, by the maximum principle, $\left.u\right|_{\partial \mathbb{D}}$ is an embedding transverse to the characteristic foliation $\tilde{S}_{\xi}^{t}$. The leaf $\tilde{\ell}_{k}^{t}$ of this characteristic foliation is given by the transverse intersection of $\tilde{S}^{t}$ and the disc $\tilde{Q}_{k}$. This implies that $\left.u\right|_{\partial \mathbb{D}}$ is transverse to $\tilde{Q}_{k}$.

For each $k \in\{0,1,2\}$, the tangent vector $\eta^{k}:=\left.(d / d \tau)\right|_{\tau=0}\left(u \circ m_{\tau}^{k}\right)$ maps, under the differential of the evaluation map, to $\left(\eta^{k}(1), \eta^{k}(i), \eta^{k}(-1)\right)$. By what we just said, the tangent vector $\eta^{k}\left(i^{k}\right)$ is transverse to $\tilde{Q}_{k} \subset S^{3}$, and $\eta^{k}\left(i^{l}\right)=0$ for $l \neq k$. This proves the lemma.

We now begin with a systematic description of the analytic setting in which we want to formulate the transversality results that will imply Proposition 7.1 We work with discs of all levels in $(-1,1)$, although it would suffice to restrict to a slightly smaller interval, since Bishop discs of level $t$ with $|t| \geq \sqrt{1-\delta}$ are standard.

By the Sobolev embedding theorem, each equivalence class of maps of class $W^{1, p}$ has a continuous representative for $1 \cdot p>\operatorname{dim}_{\mathbb{R}} \mathbb{D}=2$. We think of this representative when we speak simply of a map of class $W^{1, p}$. This allows us to introduce the following spaces.

Notation. Let $\mathcal{C} \subset W^{1, p}\left(\mathbb{D}, \mathbb{R}^{4}\right)$ be the subset of maps $u:(\mathbb{D}, \partial \mathbb{D}) \rightarrow\left(\mathbb{R}^{4}, S^{3} \backslash K\right)$ such that $\left.u\right|_{\partial \mathbb{D}}: \partial \mathbb{D} \rightarrow S^{3} \backslash K \simeq S^{1}$ lies in the homotopy class of $\left.u_{0}^{0}\right|_{\partial \mathbb{D}}$, where $u_{0}^{0}$ is one of the standard Bishop discs.

Let $\mathcal{B} \subset \mathcal{C}$ be the subset of maps $u$ such that $\left.u\right|_{\partial \mathbb{D}}$ maps $\partial \mathbb{D}$ into a punctured sphere $\tilde{S}^{t} \backslash\left\{q_{ \pm}^{t}\right\}$ for some $t \in(-1,1)$.

Observe that $\widetilde{\mathcal{W}}_{\varphi}=\{u \in \mathcal{B}: \bar{\partial} u=0\}$. Contrary to appearances, the equation $\bar{\partial} u=0$ on $\mathcal{B}$ is not a linear one, since the boundary conditions are not linear.

Proposition 7.3. The spaces $\mathcal{B}$ and $\mathcal{C}$ are Banach manifolds.

Proof. We begin with $\mathcal{C}$. Here we just give the main points; for more details see 32]. First we consider $W^{1, p}$-maps $u:(\mathbb{D}, \partial \mathbb{D}) \rightarrow\left(\mathbb{R}^{4}, S^{3}\right)$. Choose a metric on $\mathbb{R}^{4}$ for which $S^{3}$ is totally geodesic. Then the composition of a section of $u^{*}\left(T \mathbb{R}^{4}, T S^{3}\right)$ with the exponential map for this metric will be a map $(\mathbb{D}, \partial \mathbb{D}) \rightarrow\left(\mathbb{R}^{4}, S^{3}\right)$. This can be used to show that we obtain a Banach manifold modelled on the tangent

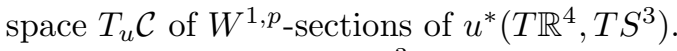

Given $u \in \mathcal{C}$, the set $S^{3} \backslash K$ is an open neighbourhood of $u(\partial \mathbb{D})$ in $S^{3}$. It then follows from the Sobolev embedding theorem that the space of maps $(\mathbb{D}, \partial \mathbb{D}) \rightarrow$ $\left(\mathbb{R}^{4}, S^{3} \backslash K\right)$ is an open subset of the previous Banach manifold. The space $\mathcal{C}$, where 
we impose an additional homotopical condition, consists of a connected component of this open subset.

We now turn to $\mathcal{B}$. Recall that the 2-spheres $S^{t} \subset S^{3}$ are the level sets of the function $H$, given by projection onto the $y_{2}$-coordinate. Write $\widetilde{H}:=H \circ \varphi^{-1}$; then the spheres $\widetilde{S}^{t}=\varphi\left(S^{t}\right)$ are the level sets of $\widetilde{H}$. This means that $\mathcal{B}$ is the inverse image of the set of constant functions under the smooth map

$$
\begin{aligned}
\Psi: \quad \mathcal{C} & \longrightarrow C^{0}(\partial \mathbb{D},(-1,1)) \\
u & \left.\longmapsto \widetilde{H} \circ u\right|_{\partial \mathbb{D}} .
\end{aligned}
$$

(Beware that a $W^{k, p}$-function loses derivatives, in general, when restricted to the boundary, see [22, p. 522].)

In order to prove that $\mathcal{B}$ is a submanifold of $\mathcal{C}$, we need to verify that $\Psi$ is transverse to the subspace of constant functions in $C^{0}(\partial \mathbb{D},(-1,1))$. Write $\mathbb{R}$ for the tangent space to this subspace. Then the condition to be verified is that for $u \in \mathcal{B}$ the composite map

$$
\Psi^{\prime}: T_{u} \mathcal{C} \stackrel{T_{u} \Psi}{\longrightarrow} T_{\Psi(u)} C^{0}(\partial \mathbb{D},(-1,1))=C^{0}(\partial \mathbb{D}, \mathbb{R}) \longrightarrow C^{0}(\partial \mathbb{D}, \mathbb{R}) / \mathbb{R}
$$

is surjective and its kernel splits, cf. [19, Proposition II.2.4].

(i) We show that $T_{u} \Psi$ is surjective (for all $u \in \mathcal{C}$ ); this obviously implies surjectivity of $\Psi^{\prime}$. Choose an auxiliary Riemannian metric $\langle.,$.$\rangle on S^{3}$. Then the differential $T_{u} \Psi$ sends a tangent vector $\eta \in T_{u} \mathcal{C}$ to

$$
T_{u} \Psi(\eta)=\left\langle\left.\nabla \widetilde{H} \circ u\right|_{\partial \mathbb{D}},\left.\eta\right|_{\partial \mathbb{D}}\right\rangle,
$$

which is a real-valued function on $\partial \mathbb{D}$.

Let $f_{0} \in C^{0}(\partial \mathbb{D}, \mathbb{R})$ be given. Poisson's formula for the ball, cf. [8, gives the unique solution $\eta_{0}$ of the boundary value problem

$$
\Delta \eta_{0}=0,\left.\quad \eta_{0}\right|_{\partial \mathbb{D}}=\left.f_{0} \cdot \frac{\nabla \widetilde{H}}{\|\nabla \widetilde{H}\|^{2}} \circ u\right|_{\partial \mathbb{D}}
$$

with $\eta_{0}: \mathbb{D} \rightarrow \mathbb{R}^{4}$ continuous on $\mathbb{D}$ and smooth in the interior of $\mathbb{D}$. Then $\eta_{0} \in T_{u} \mathcal{C}$ and $T_{u} \Psi\left(\eta_{0}\right)=f_{0}$.

(ii) Define $\mathcal{H} \subset T_{u} \mathcal{C}$ as the space of solutions of the boundary value problem (P) corresponding to functions $f_{0} \in C^{0}(\partial \mathbb{D}, \mathbb{R})$ with $f_{0}(1)=0$. We claim that $\mathcal{H}$ is complementary to $\operatorname{ker} \Psi^{\prime}$ in the sense of Banach spaces, i.e. $\mathcal{H}$ is closed in $T_{u} \mathcal{C}$, and $\operatorname{ker} \Psi^{\prime} \oplus \mathcal{H}=T_{u} \mathcal{C}$.

Given $\eta \in T_{u} \mathcal{C}$, define $f=T_{u} \Psi(\eta) \in C^{0}(\partial \mathbb{D}, \mathbb{R})$. Let $\eta_{0} \in \mathcal{H}$ be the solution of (P) for $f_{0}=f-f(1)$. Then $\eta$ obviously decomposes as $\eta=\left(\eta-\eta_{0}\right)+\eta_{0}$, and $\eta-\eta_{0} \in \operatorname{ker} \Psi^{\prime}$, since

$$
T_{u} \Psi\left(\eta-\eta_{0}\right)=f-(f-f(1))=f(1)
$$

is a constant function. This shows that $T_{u} \Psi=\operatorname{ker} \Psi^{\prime}+\mathcal{H}$.

If $\eta \in \operatorname{ker} \Psi^{\prime} \cap \mathcal{H}$, then $T_{u} \Psi(\eta)$ is a constant function on $\partial \mathbb{D}$ which takes the value 0 at 1 , so it is identically zero. By the uniqueness of the solution of $(\mathrm{P})$ this forces $\eta_{0}=0$. So we have a direct sum decomposition of $T_{u} \Psi$.

It remains to show that $\mathcal{H}$ is a closed subspace of $T_{u} \mathcal{C}$. Let $\left(\eta_{\nu}\right)$ be a sequence in $\mathcal{H}$ converging in the $W^{1, p}$-norm to some $\eta_{0} \in T_{u} \mathcal{C}$. By elliptic regularity [22, Theorem B.3.1], $\left(\eta_{\nu}\right)$ is a $W_{\text {loc }}^{k, p}$-Cauchy sequence on the interior of $\mathbb{D}$, and by the Sobolev embedding theorem a $C_{\text {loc }}^{k}$-Cauchy sequence for all $k \in \mathbb{N}$. It follows that 
$\eta_{0}$ is continuous on $\mathbb{D}$, smooth on the interior of $\mathbb{D}$, and it solves the boundary value problem $(\mathrm{P})$ for a suitable $f_{0} \in C^{0}(\partial \mathbb{D}, \mathbb{R})$ with $f_{0}(1)=0$. This means that $\eta_{0} \in \mathcal{H}$, so $\mathcal{H}$ is closed.

In order to prove that $\widetilde{\mathcal{W}}_{\varphi} \subset \mathcal{B}$ is a 5-dimensional submanifold, we need to show that the linearisation $D_{u}$ of $\bar{\partial}$ at $u \in \widetilde{\mathcal{W}}_{\varphi} \subset \mathcal{B}$ is a surjective Fredholm operator with a 5-dimensional kernel. (The splitting of ker $D_{u}$ is then given by a closed subspace isomorphic to the image of $D_{u}$.) For the proof of Proposition 5.2 we also have to exhibit explicit generators of this kernel. Since these are local statements, we first introduce a suitable local chart around a given Bishop disc $u \in \widetilde{\mathcal{W}}_{\varphi} \subset \mathcal{B}$ of level $t$. For an open neighbourhood $U$ of $u(\mathbb{D})$ in $\mathbb{C}^{2}$, the set $\{w \in \mathcal{B}: w(\mathbb{D}) \subset U\}$ is, by the Sobolev embedding theorem, an open neighbourhood of $u$ in $\mathcal{B}$. Our aim is to find a neighbourhood $U$ and a chart $\iota: U \longrightarrow \mathbb{C} \times \mathbb{C}$ adapted to our Fredholm problem.

To this end, choose a frame $e_{1}, e_{2}$ of $T \tilde{S}^{t}$ along $u(\partial \mathbb{D})$ with $e_{1}$ tangent to $u(\partial \mathbb{D})$, with $e_{2}$ tangent to the characteristic foliation $\tilde{S}_{\xi}^{t}$, and with $J_{0} e_{2}$ - which is tangent to $S^{3}$ but transverse to $\tilde{S}^{t}$ - pointing in the direction of increasing $\widetilde{H}$. Choose a Riemannian metric on $\mathbb{R}^{4}$ which makes $e_{1}, J_{0} e_{1}, e_{2}, J_{0} e_{2}$ an orthonormal frame along $u(\partial \mathbb{D})$. Set $e_{2}^{\prime}=e_{2} /\|\nabla \widetilde{H}\|$, where the gradient and norm are taken relative to the restriction of the chosen metric to $S^{3}$. Notice that $J_{0} e_{2}^{\prime}$ equals $\nabla \widetilde{H} /\|\nabla \widetilde{H}\|^{2}$, since both vector fields are tangent to $S^{3}$, orthogonal to $\tilde{S}^{t}$ pointing in the same direction, and have the same length. We may extend $u$ to an embedding defined on a small neighbourhood of $\mathbb{D}$ in $\mathbb{C}$. Then we find an open neighbourhood of $\mathbb{D} \times\{0\}$ in $\mathbb{C} \times \mathbb{C}$ on which the map

$$
\begin{array}{ccc}
\mathbb{C} \times \mathbb{C} & \longrightarrow & \mathbb{C}^{2} \\
\left(z_{1}, x_{2}+i y_{2}\right) & \longmapsto & \exp _{u\left(z_{1}\right)}\left(x_{2} e_{2}^{\prime}+y_{2} J_{0} e_{2}^{\prime}\right)
\end{array}
$$

defines an embedding. Let $\iota$ be the inverse map, defined on the image of that embedding. We summarise the properties of $\iota$, which are obvious from the construction, in the following lemma.

Lemma 7.4. There is a neighbourhood $U$ of $u(\mathbb{D}) \subset \mathbb{R}^{4}$ and an embedding $\iota: U \rightarrow$ $\mathbb{C} \times \mathbb{C}$ with the following properties:

(1) $\iota(u(z))=(z, 0)$ for all $z \in \mathbb{D}$.

(2) $\iota_{*} J_{0}:=T \iota \circ J_{0} \circ T \iota^{-1}=J_{0}$ on $\left.T \mathbb{R}^{4}\right|_{\mathbb{D} \times\{0\}}$.

(3) For each $z \in \partial \mathbb{D}$, the differential $T_{u(z)} \iota$ sends the tangent direction along the leaf of the characteristic foliation $\tilde{S}_{\xi}^{t}$ through $u(z)$ to $\{0\} \times \mathbb{R} \subset T_{(z, 0)} \mathbb{C}^{2}$.

(4) For each $z \in \partial \mathbb{D}$, the differential $T_{u(z)} \iota$ sends $\nabla \widetilde{H} /\|\nabla \widetilde{H}\|^{2}$ to $(0, i) \subset$ $T_{(z, 0)} \mathbb{C}^{2}$.

Thanks to this lemma we may now assume that $u$ is the inclusion $\mathbb{D} \rightarrow \mathbb{D} \times\{0\} \subset$ $\mathbb{C} \times \mathbb{C}$, at the cost of replacing $J_{0}$ by a complex structure $J$ that varies from point to point, but coincides with $J_{0}$ along $\mathbb{D} \times\{0\}$.

So the linearisation $D_{u}$ of the Cauchy-Riemann operator $\bar{\partial}_{J}=\partial_{x}+J \partial_{y}$ at the point $u \in \widetilde{\mathcal{W}}_{\varphi} \subset \mathcal{B}$ is given by

$$
\begin{aligned}
D_{u}: \quad T_{u} \mathcal{B} & \longrightarrow \\
\eta & \longmapsto \bar{\partial} \eta+\left(\partial_{\eta} J\right) \partial_{y} u .
\end{aligned}
$$

This is a real linear Cauchy-Riemann operator in the sense of [22, Appendix C] and so is Fredholm. 
Proposition 7.5. The operator $D_{u}$ is onto and has a 5-dimensional kernel. This kernel is spanned by $d u(\mathfrak{a u t}(\mathbb{D}))$, where $\mathfrak{a u t}(\mathbb{D})$ denotes the 3 -dimensional Lie algebra of infinitesimal holomorphic automorphisms of $\mathbb{D}$, and two smooth sections $\sigma, \tau$ of $u^{*}\left(T \mathbb{C}^{2}, T \tilde{S}^{t}\right)$ and $u^{*}\left(T \mathbb{C}^{2}, T S^{3}\right)$, respectively, with the following properties:

(1) For $z \in\{1, i,-1\}$, the vector $\sigma(z) \in T_{u(z)} \tilde{S}^{t}$, is tangent to the characteristic foliation $\tilde{S}_{\xi}^{t}$.

(2) The component of the vector field $\left.\tau\right|_{\partial \mathbb{D}}$ orthogonal to $T \tilde{S}^{t}$ is a positive constant multiple of $\nabla \widetilde{H} /\|\nabla \widetilde{H}\|^{2}$. For $u \in \mathcal{W} \subset \widetilde{\mathcal{W}}$ we may assume in addition that for $k=0,1,2$ the vector $\tau\left(i^{k}\right) \in T_{u\left(i^{k}\right)} S^{3}$ is tangent to $\tilde{Q}_{k}$.

(3) For each $z \in \mathbb{D}$ the vectors $\sigma(z)$ and $\tau(z)$ span the complement of $d u\left(T_{z} \mathbb{D}\right)$ in $T_{u(z)} \mathbb{R}^{4}$.

Remarks. (1) The notation $\sigma$ and $\tau$ is meant to be mnemonic. The component of the vector field $\left.\sigma\right|_{\partial \mathbb{D}}$ orthogonal to $u(\partial \mathbb{D})$ points along a leaf of the characteristic foliation $\tilde{S}_{\xi}^{t}$ in the direction of the positive $s$-parameter; the vector field $\left.\tau\right|_{\partial \mathbb{D}}$ corresponds to an infinitesimal shift of $t$-level spheres.

(2) Conditions (1) and (2) in the proposition guarantee that for $u \in \mathcal{W}$ the tangent vectors $\sigma$ and $\tau$ lie in the kernel of the differential of the evaluation map $\mathrm{ev}_{1, i,-1}$, since

$$
T_{u} \mathrm{ev}_{1, i,-1}(\eta)=(\eta(1), \eta(i), \eta(-1)) .
$$

So $\sigma$ and $\tau$ may be regarded as elements of the tangent space $T_{u} \mathcal{W}$.

Proof of Proposition 7.5. In our local model around $u$, the elements $\eta$ of the tangent space $T_{u} \mathcal{B}$ can be written as sections of the form $\left(\eta^{\|}, \eta^{\perp}\right) \in W^{1, p}(\mathbb{D}, \mathbb{C} \oplus \mathbb{C})$. The condition that $\eta(z)$ be tangent to $S^{3}$ for $z \in \partial \mathbb{D}$ translates into

$$
\eta^{\|}(z) \in i z \mathbb{R} \text { for } z \in \partial \mathbb{D} \text {. }
$$

The real part of $\eta^{\perp}(z)$ for $z \in \partial \mathbb{D}$ corresponds to the tangential direction of a leaf of the characteristic foliation $\tilde{S}_{\xi}^{t}$; the imaginary part, to $\nabla \widetilde{H} /\|\nabla \widetilde{H}\|^{2}$. The flow of the latter vector field preserves the level spheres of $\widetilde{H}$. Since the Banach manifold $\mathcal{B}$ consists of discs whose boundary lies in such a level sphere, we have the boundary condition

$$
\operatorname{Im} \eta^{\perp}(z)=\text { const. for } z \in \partial \mathbb{D} .
$$

Our real linear Cauchy-Riemann operator $D_{u}$ now takes the form

$$
\begin{array}{cccc}
D_{u}: & T_{u} \mathcal{B} & \longrightarrow & L^{p}(\mathbb{D}, \mathbb{C} \oplus \mathbb{C}) \\
\left(\eta^{\|}, \eta^{\perp}\right) & \longmapsto & \left(\bar{\partial} \eta^{\|}+A \eta^{\perp},(\bar{\partial}+B) \eta^{\perp}\right),
\end{array}
$$

where $A$ and $B$ are smooth maps from $\mathbb{D}$ into the real endomorphisms of $\mathbb{C}=\mathbb{R}^{2}$. Elliptic regularity once again gives us smoothness of the tangent vectors that lie in $\operatorname{ker} D_{u} \subset T_{u} \mathcal{B}$.

(i) When the boundary condition on $\eta^{\perp}$ is strengthened to $\operatorname{Im} \eta^{\perp}=0$ for $z \in \partial \mathbb{D}$ - this corresponds to tangent vectors along the submanifold of $\mathcal{B}$ of maps of level $t$ - , the vector $\left(\eta^{\|}(z), \eta^{\perp}(z)\right)$ lies in the totally real subspace $i z \mathbb{R} \oplus \mathbb{R} \subset \mathbb{C} \oplus \mathbb{C}$ for each $z \in \partial \mathbb{D}$. This means we are dealing with a Riemann-Roch boundary value problem in the sense of [22, p. 544]. The Maslov index of the bundle pair describing the boundary condition equals 2 , so by [22, Theorem C.1.10] the operator $D_{u}$ is a surjective Fredholm operator of index 4 . A fortiori, $D_{u}$ is onto when the boundary condition is relaxed to $\operatorname{Im} \eta^{\perp}(z)=$ const. for $z \in \partial \mathbb{D}$. 
(ii) We first consider tangent vectors of the form $\left(\eta^{\|}, 0\right)$. Such a tangent vector lies in ker $D_{u}$ precisely when $\eta^{\|}$is holomorphic. By expanding $\eta^{\|}$into a power series on $\mathbb{D}$, it is easy to see that the condition $\eta^{\|}(z) \in i z \mathbb{R}$ for $z \in \partial \mathbb{D}$ is equivalent to $\eta^{\|}$being of the form

$$
\eta^{\|}(z)=a+i b z-\bar{a} z^{2}
$$

for some $a \in \mathbb{C}$ and $b \in \mathbb{R}$, cf. [14, p. 75]. It is a nice calculation to verify that these are indeed the infinitesimal Möbius transformations of $\mathbb{D}$. In other words, the subspace of $\operatorname{ker} D_{u}$ of elements of the form $\left(\eta^{\|}, 0\right)$ is the 3-dimensional space $\mathfrak{a u t}(\mathbb{D}) \times\{0\}$.

(iii) If $\left(\eta^{\|}, \eta^{\perp}\right)$ is an element of ker $D_{u}$, then in particular $(\bar{\partial}+B) \eta^{\perp}=0$, and for all $z \in \partial \mathbb{D}$ we have $\eta^{\perp}(z)=c i$ for some real constant $c$; we call such an $\eta^{\perp}$ admissible. Any admissible $\eta^{\perp}$ can be written as $\eta^{\perp}=w+c i$, where $w$ is a smooth solution of the Riemann problem

$$
(\bar{\partial}+B) w=-c B i, \quad w(z) \in \mathbb{R} \text { for } z \in \partial \mathbb{D} .
$$

(Beware that on the right-hand side of this equation, $B$ is a real endomorphism acting on the vector $i \in \mathbb{C}=\mathbb{R}^{2}$.) Conversely, any solution $w$ of $\left(\mathrm{R}_{c}\right)$ gives rise to an admissible $\eta^{\perp}=w+c i$.

The boundary condition in $\left(\mathrm{R}_{c}\right)$ defines a bundle pair of Maslov index 0 , so by the Riemann-Roch theorem [22, Theorem C.1.10] the operator $\bar{\partial}+B$ is a surjective Fredholm operator of index 1 . Choose a non-trivial solution $w_{0}$ of $\left(\mathrm{R}_{0}\right)$ and any solution $w_{1}$ of $\left(\mathrm{R}_{1}\right)$. If $w_{c}$ is a solution of $\left(\mathrm{R}_{c}\right)$, then $w_{c}-c w_{1}$ is a solution of $\left(\mathrm{R}_{0}\right)$. It follows that the space of pairs $\left(w_{c}, c\right)$ with $w_{c}$ a solution of $\left(\mathrm{R}_{c}\right)$ is 2-dimensional, spanned by $\left(w_{0}, 0\right)$ and $\left(w_{1}, 1\right)$. We conclude that the space of admissible $\eta^{\perp}$ is spanned by $\eta_{0}^{\perp}:=w_{0}$ and $\eta_{1}^{\perp}:=w_{1}+i$.

(iv) Next we want to show that any admissible $\eta^{\perp}$ is in fact the component of a tangent vector $\left(\eta^{\|}, \eta^{\perp}\right)$ that lies in $\operatorname{ker} D_{u}$. Thus, given an admissible $\eta^{\perp}$, we are now looking for a solution $\eta^{\|}: \mathbb{D} \rightarrow \mathbb{C}$ of the equation $\bar{\partial} \eta^{\|}=-A \eta^{\perp}$, with $\eta^{\|}(z) \in i z \mathbb{R}$ for all $z \in \partial \mathbb{D}$. This boundary condition defines a bundle pair of Maslov index 2, so again by the Riemann-Roch theorem [22, Theorem C.1.10] the operator $\bar{\partial}$ is a surjective Fredholm operator of index 3. By (ii) we find a unique solution $\eta^{\|}$with prescribed values in $i z \mathbb{R}$ at the points $z=1, i,-1$.

Starting with $\eta_{0}^{\perp}$ or $\eta_{1}^{\perp}$ from (iii) we obtain the tangent vectors $\sigma$ and $\tau$ that satisfy conditions (1) and (2), respectively, of the proposition. The $\eta^{\|}$-component of $\sigma$ has to vanish at the points $1, i,-1$, since the real part of $\eta^{\perp}$ corresponds to the direction of the characteristic foliation $\tilde{S}_{\xi}^{t}$ along $\partial \mathbb{D}$; for $u \in \mathcal{W}$ the $\eta^{\|}$-component $\eta_{1}^{\|}$of $\tau$ is determined by the condition

$$
\eta_{1}^{\|}\left(i^{k}\right)+\eta_{1}^{\perp}\left(i^{k}\right) \in T_{u\left(i^{k}\right)} \tilde{Q}_{k}, \quad k=0,1,2 .
$$

(v) It remains to verify condition (3). For this we need to show that an $\mathbb{R}$-linear combination $\lambda_{0} w_{0}+\lambda_{1}\left(w_{1}+i\right)$ that vanishes at some point in $\mathbb{D}$ has to be trivial. The 1-dimensional Carleman similarity principle [13, Corollary 13] guarantees that any solution $\eta^{\perp}$ of the equation $(\bar{\partial}+B) \eta^{\perp}=0$ can be written in the form $\eta^{\perp}=e^{g} f$, where $f, g: \mathbb{D} \rightarrow \mathbb{C}$ are continuous functions with $f$ holomorphic in the interior of $\mathbb{D}$ and $g(\partial \mathbb{D}) \subset \mathbb{R}$. Now write $\lambda_{0} w_{0}+\lambda_{1}\left(w_{1}+i\right)$ in this form. We need to show that $\lambda_{0}=\lambda_{1}=0$ if $f$ has any zeros. 
Suppose there is a point $z_{0} \in \mathbb{D}$ with $f\left(z_{0}\right)=0$. If $z_{0} \in \partial \mathbb{D}$, then $\lambda_{1}=0$. If $z_{0}$ lies in the interior of $\mathbb{D}$, we may assume after a Möbius transformation of $\mathbb{D}$ that $z_{0}=0$. The function $f$, being continuous on $\mathbb{D}$ and holomorphic on $\operatorname{Int} \mathbb{D}$, satisfies the mean value property

$$
0=f(0)=\frac{1}{2 \pi} \int_{0}^{2 \pi} f\left(e^{i \theta}\right) d \theta
$$

By considering the imaginary part of this equation we see once again that $\lambda_{1}=0$.

This means that the function $\operatorname{Im} f$, which is harmonic on the interior of $\mathbb{D}$ and continuous on $\mathbb{D}$, is zero on $\partial \mathbb{D}$, and hence zero on $\mathbb{D}$ by the maximum principle. So the holomorphic function $f$ is constant, and since it vanishes in $z_{0}$ it is the zero function, which implies $\lambda_{0}=0$.

Proof of Proposition 5.1. The moduli space $\mathcal{W}_{\varphi}^{\delta}$ has been established as a smooth compact surface. So the image of the evaluation map ev 1 will likewise be compact. The differential $T_{u} \mathrm{ev}_{1}$ has full rank by the preceding proposition, since $T_{u} \mathrm{ev}_{1}(\eta)=$ $\eta(1)$. So the image of ev 1 will also be open in $\tilde{Q}^{\delta}$, which means that $e_{1}$ is in fact surjective. Injectivity of $\mathrm{ev}_{1}$ follows from Proposition 4.5.

Proof of Proposition 5.2. Since the bidisc $\mathbb{D} \times \mathbb{D}_{1-\delta}$ is a compact manifold, we need only show that $F^{\delta}$ is an injective immersion. Injectivity follows from all Bishop discs being embedded and mutually disjoint. That the differential of $F^{\delta}$ has full rank is an immediate consequence of Proposition 7.5 (3).

\section{TOPOLOGICAL INTERSECTION OF DisCS}

In Section 9 below we want to establish results about the positivity of intersections of holomorphic discs that are instrumental for proving Proposition 4.5. The present section deals with the topological preliminaries. The main results in this and the following section can be found in [31, but we feel that a more self-contained presentation is appropriate in our context. Some subtle issues concerning intersection points accumulating at the boundary (see Proposition 9.1 below) have not been addressed in the cited paper. Intersection properties of holomorphic discs are also discussed in [30, Section 4.4].

Let $W$ be an oriented 4-dimensional manifold with boundary $M=\partial W$, and $\Sigma \subset M$ an embedded oriented surface. Our aim in this section is to define an intersection pairing on the relative homotopy group $\pi_{2}(W, \Sigma)$. Elements in this group are based homotopy classes of $C^{0}$-maps $(\mathbb{D}, \partial \mathbb{D}) \rightarrow(W, \Sigma)$.

Definition. Let $\mathcal{D}$ be the space of $C^{0}$-maps $(\mathbb{D}, \partial \mathbb{D}) \rightarrow(W, \Sigma)$. A disc $u \in \mathcal{D}$ is called admissible if

(1) $u$ is smooth,

(2) $u$ maps the interior of $\mathbb{D}$ into the interior of $W$,

(3) $\left.u\right|_{\partial \mathbb{D}}$ is an immersion, and

(4) $u$ is transverse to $M$.

Write $\mathcal{A} \subset \mathcal{D}$ for the subset of admissible discs.

Observe that in the case of $W=D^{4}, M=S^{3}$, and $\Sigma \subset S^{3}$ a totally real surface, any holomorphic disc $u \in \mathcal{D}$ is admissible thanks to regularity and the maximum principle. 
The following is a consequence of standard approximation and general position results in differential topology, see [12, Chapter 2].

Proposition 8.1. The subset of admissible discs $\mathcal{A}$ is dense in $\mathcal{D}$.

The set of all intersection points of two discs $u_{1}, u_{2} \in \mathcal{D}$ is

$$
S\left(u_{1}, u_{2}\right):=\left\{\left(z_{1}, z_{2}\right) \in \mathbb{D} \times \mathbb{D}: u_{1}\left(z_{1}\right)=u_{2}\left(z_{2}\right)\right\} .
$$

For the purpose of defining an intersection number of $u_{1}$ and $u_{2}$ we distinguish the following subsets of $S\left(u_{1}, u_{2}\right)$.

Definition. The set of interior intersection points of $u_{1}, u_{2} \in \mathcal{D}$ is

$$
S_{\text {Int }}\left(u_{1}, u_{2}\right):=S\left(u_{1}, u_{2}\right) \cap(\operatorname{Int} \mathbb{D} \times \operatorname{Int} \mathbb{D}) .
$$

The set of boundary intersection points is

$$
S_{\partial}\left(u_{1}, u_{2}\right):=S\left(u_{1}, u_{2}\right) \cap(\partial \mathbb{D} \times \partial \mathbb{D}) .
$$

By the requirement that an admissible disc map Int $\mathbb{D}$ to the interior of $W$, there can be no mixed intersection points, hence

$$
S\left(u_{1}, u_{2}\right)=S_{\text {Int }}\left(u_{1}, u_{2}\right) \sqcup S_{\partial}\left(u_{1}, u_{2}\right) .
$$

Definition. We say that two admissible $\operatorname{discs} u_{1}, u_{2} \in \mathcal{A}$ intersect nicely if $S\left(u_{1}, u_{2}\right)$ is a finite set of isolated intersection points, and if for any $\left(z_{1}, z_{2}\right) \in$ $S_{\partial}\left(u_{1}, u_{2}\right)$ the two 2-planes

$$
d u_{1}\left(T_{z_{1}} \mathbb{D}\right), d u_{2}\left(T_{z_{2}} \mathbb{D}\right) \subset T_{u_{1}\left(z_{1}\right)} W=T_{u_{2}\left(z_{2}\right)} W
$$

either are transverse or coincide. Write $\mathcal{P} \subset \mathcal{A} \times \mathcal{A}$ for the set of pairs of nicely intersecting discs.

Notice that the second condition of this definition is automatically satisfied for any two holomorphic discs.

Proposition 8.2. The set $\mathcal{P}$ of pairs of nicely intersecting discs is dense in $\mathcal{D} \times \mathcal{D}$.

Proof. By Proposition 8.1] it suffices to show that $\mathcal{P}$ is dense in $\mathcal{A} \times \mathcal{A}$. Let $u_{1}, u_{2} \in \mathcal{A}$ be given. We are going to find an arbitrarily $C^{\infty}$-small isotopic perturbation of $u_{1}$ to a new disc intersecting $u_{2}$ nicely. The necessary differential topological methods are supplied by 12 .

In a first step, we find an arbitrarily $C^{\infty}$-small homotopy of $\left.u_{1}\right|_{\partial \mathbb{D}}$ to an immersion transverse to $\left.u_{2}\right|_{\partial \mathbb{D}}$. By sweeping out this homotopy over a collar neighbourhood of $M$ in $W$, this extends to a homotopy of $u_{1}$. We continue to write $u_{1}$ for the new map after this and each of the following perturbations.

So after this first step the maps $\left.u_{1}\right|_{\partial \mathbb{D}}$ and $\left.u_{2}\right|_{\partial \mathbb{D}}$ are transverse immersions of the circle into $\Sigma$, but $u_{1}$ and $u_{2}$ may fail to be transverse along $\partial \mathbb{D}$. This, however, can only happen at one of the finitely many intersection points of $u_{1}(\partial \mathbb{D})$ and $u_{2}(\partial \mathbb{D})$. In a collar neighbourhood $(-\varepsilon, 0] \times M$ of $M$ in $W$ one can define a $C^{\infty}$-small perturbation of $u_{1}$, supported in this collar neighbourhood and relative to $\partial \mathbb{D}$, in a direction transverse to $\Sigma \subset M$.

After this second perturbation we may assume that $u_{1}$ and $u_{2}$ are transverse near $\partial \mathbb{D}$. Then $S_{\text {Int }}\left(u_{1}, u_{2}\right)$ does not have any accumulation points at the boundary, and a further perturbation of $u_{1}$ outside a neighbourhood of $\partial \mathbb{D}$ in $\mathbb{D}$ gives us a nicely intersecting pair $\left(u_{1}, u_{2}\right)$. 
Remark. The proof shows that even the subset of pairs $\left(u_{1}, u_{2}\right)$ with transverse intersections only is dense in $\mathcal{A} \times \mathcal{A}$.

As we shall see in the next section, two distinct holomorphic discs will intersect nicely, but not - a priori - transversely. For this reason, the above proposition has been phrased in terms of nicely intersecting discs. We now wish to define an intersection number for such discs by assigning an intersection multiplicity to each isolated intersection point, cf. [21], 22, Definition E.2.1]. We distinguish three types of intersections, where in each case we write $p:=u_{1}\left(z_{1}\right)=u_{2}\left(z_{2}\right)$ for $\left(z_{1}, z_{2}\right) \in S\left(u_{1}, u_{2}\right)$.

(i) Interior intersections. Given $\left(z_{1}, z_{2}\right) \in S_{\text {Int }}\left(u_{1}, u_{2}\right)$, we define the intersection multiplicity $\left(u_{1} \cdot u_{2}\right)_{\left(z_{1}, z_{2}\right)}$ as in the cited references. Choose neighbourhoods of $p$ in $u_{1}(\mathbb{D})$ and $u_{2}(\mathbb{D})$ that intersect in $p$ only. Then perturb the discs inside these neighbourhoods, keeping either neighbourhood disjoint from the boundary of the other, so as to achieve transversality of intersection. Then count the intersection points with sign according to the orientation. Notice that for the definition of this intersection multiplicity the maps $u_{1}$ and $u_{2}$ need not be smooth; it suffices that the intersection points be isolated.

(ii) Transverse boundary intersections. Here the intersection multiplicity can be defined as for an interior transverse intersection point. If $\left(z_{1}, z_{2}\right) \in S_{\partial}\left(u_{1}, u_{2}\right)$ is a transverse intersection point, the Whitney sum

$$
d u_{1}\left(T_{z_{1}} \mathbb{D}\right) \oplus d u_{2}\left(T_{z_{2}} \mathbb{D}\right)
$$

is isomorphic to $T_{p} W$. The intersection multiplicity $\left(u_{1} \cdot u_{2}\right)_{\left(z_{1}, z_{2}\right)}$ equals \pm 1 , depending on whether this isomorphism is orientation preserving or reversing.

(iii) Tangential boundary intersections (cf. [31, 30]). Let $\left(z_{1}, z_{2}\right) \in S_{\partial}\left(u_{1}, u_{2}\right)$ be a tangential intersection point, i.e. $d u_{1}\left(T_{z_{1}} \mathbb{D}\right)=d u_{2}\left(T_{z_{2}} \mathbb{D}\right)$. Since $u_{1}$ and $u_{2}$ are transverse to $M$ from the same side, we can write $u_{2}(\mathbb{D})$ locally as a graph over $u_{1}(\mathbb{D})$. It is not possible, in general, to give a homotopy-invariant definition of an intersection multiplicity when this 'same side' condition is dropped, see [30, Example 4.4.7]. We write the local model for

$$
p \in \Sigma \subset M=\partial W \subset W
$$

as

$$
0 \in \mathbb{R} \times \mathbb{R} \subset \mathbb{R} \times \mathbb{C} \subset \mathbb{H} \times \mathbb{C} .
$$

(This complex model is chosen for convenience of notation only; we do not consider holomorphic discs yet.) Moreover, we may assume that the local model has been selected in such a way that the local description of $u_{1}$ and $u_{2}$ is of the form

$$
(\mathbb{H}, \mathbb{R}) \longrightarrow(\mathbb{H} \times \mathbb{C}, \mathbb{R} \times \mathbb{R})
$$

with

$$
u_{1}(z)=(z, 0) \text { and } u_{2}(z)=(z, h(z)) .
$$

Now extend $u_{1}$ and $u_{2}$ by Schwarz reflection to continuous maps

$$
(\mathbb{C}, \mathbb{R}) \longrightarrow(\mathbb{C} \times \mathbb{C}, \mathbb{R} \times \mathbb{R}) .
$$

For $u_{1}$ this simply means extending the definition $u_{1}(z)=(z, 0)$ to $z \in \mathbb{C}$; for $u_{2}$ we set

$$
u_{2}(z)=(z, \overline{h(\bar{z})}) \text { for } \operatorname{Im} z<0 .
$$


Up to homotopy, the definition of this extension is independent of the choice of local coordinates, and so the intersection multiplicity $\left(u_{1} \cdot u_{2}\right)_{\left(z_{1}, z_{2}\right)}$ is well defined as the intersection multiplicity in the sense of (i) of these extended maps.

The definition of the intersection multiplicity as in (iii) can also be used for transverse boundary intersections, in which case it coincides with that in (ii). Just as in (i), this allows us to assign a multiplicity to any isolated intersection point of discs that are merely continuous.

The following example will motivate the definition of the overall intersection number of a nicely intersecting pair $\left(u_{1}, u_{2}\right)$.

Example. Consider the maps $u_{1}(z)=(z, 0)$ and $u_{2}(z)=\left(z, z^{3}\right), z \in \mathbb{H}$, in the local model just described. The intersection point $(0,0)$ is a tangential boundary intersection of multiplicity 3 . We can perturb the extended $u_{2}$ within the class of maps symmetric with respect to Schwarz reflection to $u_{2}^{\varepsilon}(z)=\left(z, z^{3}-\varepsilon^{3}\right), z \in \mathbb{C}$, with $\varepsilon>0$, say. Then $u_{1}$ and $u_{2}^{\varepsilon}$ intersect in the three points $\varepsilon \exp (2 \pi i k / 3), k=$ $0,1,2$, all of multiplicity 1 . One of these intersection points still sits on the original boundary, now as a transverse intersection point. The two other intersection points correspond to each other under Schwarz reflection; only one of them lies inside the actual discs $u_{1}, u_{2}^{\varepsilon}$, i.e. in the non-extended domain of definition $\mathbb{H}$ (in the local model) of these discs.

Notice that we have used the condition that $u_{1}, u_{2}$ map $\partial \mathbb{D}$ to $\Sigma \subset M$ in an essential way for the extension of $u_{1}, u_{2}$ via Schwarz reflection. The more obvious way of extending $u_{1}, u_{2}$ by doubling along $M$, which would require only that $\partial \mathbb{D}$ map to $M$, would lead to intersection points on the boundary of multiplicity 0 , and pairs of intersection points (related by the symmetry) of opposite multiplicity.

Definition. The intersection number of a pair $\left(u_{1}, u_{2}\right) \in \mathcal{P}$ of nicely intersecting admissible discs is

$$
u_{1} \bullet u_{2}:=2 \sum_{S_{\text {Int }}\left(u_{1}, u_{2}\right)}\left(u_{1} \cdot u_{2}\right)_{\left(z_{1}, z_{2}\right)}+\sum_{S_{\partial}\left(u_{1}, u_{2}\right)}\left(u_{1} \cdot u_{2}\right)_{\left(z_{1}, z_{2}\right)},
$$

where the sums are meant to be taken over the pairs $\left(z_{1}, z_{2}\right)$ of points in the sets $S_{\text {Int }}\left(u_{1}, u_{2}\right)$ and $S_{\partial}\left(u_{1}, u_{2}\right)$, respectively.

As observed by Ye [31, Lemma 7.2], this intersection number is a homotopy invariant.

Proposition 8.3. The intersection number $u_{1} \bullet u_{2}$ depends only on the homotopy classes $\left[u_{1}\right],\left[u_{2}\right] \in \pi_{2}(W, \Sigma)$.

Our proof follows a route slightly different from Ye's. Before we turn to the formal argument, we illustrate by an example the kind of deformations we shall employ.

Example. We can picture transverse boundary intersections in the same local model used above to describe tangential boundary intersections. We always take $u_{1}(z)=(z, 0)$. The two maps $u_{2}(z)=(z, z)$ and $u_{2}^{*}(z)=(z,-z)$ give local models for a transverse boundary intersection, both of the same sign, i.e. $\left(u_{1} \cdot u_{2}\right)_{(0,0)}=$ $\left(u_{1} \cdot u_{2}^{*}\right)_{(0,0)}$. However, the signs of the intersection of the boundary curves in $\mathbb{R} \times \mathbb{R}$ are different: $\left(\left.\left.u_{1}\right|_{\mathbb{R}} \cdot u_{2}\right|_{\mathbb{R}}\right)_{(0,0)}=-\left(\left.\left.u_{1}\right|_{\mathbb{R}} \cdot u_{2}^{*}\right|_{\mathbb{R}}\right)_{(0,0)}$. 
Choose some small $\varepsilon>0$ and let $h_{2}: \mathbb{R}_{0}^{+} \rightarrow \mathbb{R}$ be a smooth function with $h_{2}(y)=y$ for $y \in[0, \varepsilon)$ and $h_{2}(y)=-y$ for $y \geq 2 \varepsilon$, with a single further zero at $3 \varepsilon / 2$, where $h_{2}^{\prime}(3 \varepsilon / 2)<0$. Then the map $z=x+i y \mapsto\left(z,-x+i h_{2}(y)\right)$ coincides with $u_{2}^{*}$ outside a small neighbourhood of the boundary, but near the boundary it looks like $z \mapsto(z,-\bar{z})$.

Suppose for argument's sake that the orientations in the model had been chosen in such a way that $\left(u_{1} \cdot u_{2}^{*}\right)_{(0,0)}=1$, but $\left(\left.\left.u_{1}\right|_{\mathbb{R}} \cdot u_{2}^{*}\right|_{\mathbb{R}}\right)_{(0,0)}=-1$. After the described perturbation near the boundary, both intersections are negative. The new transverse intersection point in the interior at $z=3 \varepsilon i / 2$ is positive, so the intersection number $u_{1} \bullet u_{2}^{*}$ is not affected by this perturbation.

This example shows the following. Let $u_{1}, u_{2}$ be a pair of transversely intersecting admissible discs. Then we may perturb $u_{2}$ near the boundary in such a way that the intersection number $u_{1} \bullet u_{2}$ remains unchanged, but the intersection multiplicity at each boundary intersection point equals the intersection multiplicity of the boundary curves in $\Sigma$ at that point.

Observe that the two models where the intersection multiplicities coincide in the way described are $z \mapsto(z, z)$ and $z \mapsto(z,-\bar{z})$. In either of these models the imaginary part of the second coordinate equals $y$, which means that both models depict discs on the same side of $\Sigma \subset M$ in a collar neighbourhood of $M=\partial W$ in $W$.

Proof of Proposition 8.3. Let $u_{1}^{0}$ and $u_{1}^{1}$ be admissible discs with $\left[u_{1}^{0}\right]=\left[u_{1}^{1}\right] \in$ $\pi_{2}(W, \Sigma)$, either of which nicely intersects a further admissible disc $u_{2}$. We need to show that $u_{1}^{0} \bullet u_{2}=u_{1}^{1} \bullet u_{2}$.

In case there are tangential boundary intersections, we make a small homotopic perturbation of $u_{1}^{0}, u_{1}^{1}$ as in the first of our examples so as to get transverse intersections only. This does not affect the intersection number. Next, we perform a homotopy as in the second example to adjust the signs of the boundary intersections. We continue to write $u_{1}^{0}, u_{1}^{1}$ for these discs.

Since $u_{1}^{0}$ and $u_{1}^{1}$ are homotopic, so a fortiori are the boundary curves $\left.u_{1}^{0}\right|_{\partial \mathbb{D}}$ and $\left.u_{1}^{1}\right|_{\partial \mathbb{D}}$. Hence

$$
\sum_{S_{\partial}\left(u_{1}^{0}, u_{2}\right)}\left(u_{1}^{0} \cdot u_{2}\right)_{\left(z_{1}, z_{2}\right)}=\left.\left.u_{1}^{0}\right|_{\partial \mathbb{D}} \bullet u_{2}\right|_{\partial \mathbb{D}}=\left.\left.u_{1}^{1}\right|_{\partial \mathbb{D}} \bullet u_{2}\right|_{\partial \mathbb{D}}=\sum_{S_{\partial}\left(u_{1}^{1}, u_{2}\right)}\left(u_{1}^{1} \cdot u_{2}\right)_{\left(z_{1}, z_{2}\right)},
$$

where $\bullet$ is the usual intersection product of curves on $\Sigma$.

A collar neighbourhood of $\Sigma$ in $W$ can be written globally as $(-\varepsilon, 0] \times \mathbb{R} \times \Sigma$. Given the observation before this proof, we may assume that the disc $u_{2}(\mathbb{D})$ looks like $(-\varepsilon, 0] \times\{0\} \times u_{2}(\partial \mathbb{D})$ in this collar, while the $\operatorname{discs} u_{1}^{j}, j=0,1$, look like $\left\{(-t, t, q): t \in[0, \varepsilon), q \in u_{1}^{j}(\partial \mathbb{D})\right\}$.

Now the disc $u_{1}^{0}$, a homotopy from $\left.u_{1}^{0}\right|_{\partial \mathbb{D}}$ to $\left.u_{1}^{1}\right|_{\partial \mathbb{D}}$, and the disc $u_{1}^{1}$ with reversed orientation define a map $f: S^{2} \rightarrow W$. When we push this 2 -sphere away from the boundary $\partial W$ in the direction of the tangent vector $(-1,1,0,0)$ to $(-\varepsilon, 0] \times \mathbb{R} \times \Sigma$, we remove all boundary intersection points without creating any new intersections. So the intersection number of this 2 -sphere with $u_{2}$ equals

$$
\sum_{S_{\text {Int }}\left(u_{1}^{0}, u_{2}\right)}\left(u_{1}^{0} \cdot u_{2}\right)_{\left(z_{1}, z_{2}\right)}-\sum_{S_{\text {Int }}\left(u_{1}^{1}, u_{2}\right)}\left(u_{1}^{1} \cdot u_{2}\right)_{\left(z_{1}, z_{2}\right)} .
$$


This intersection number is well defined homotopically (even homologically), i.e. there is an intersection product

$$
\bullet: H_{2}(W) \otimes H_{2}(W, \partial W) \longrightarrow \mathbb{Z},
$$

and the intersection product $[f] \bullet\left[u_{2}\right]$ is given by the intersection number above, cf. [3, Section VI.11]. However, from $\left[u_{1}^{0}\right]=\left[u_{1}^{1}\right]$ in $\pi_{2}(W, \Sigma)$ we deduce that $f$ is homotopically and hence homologically trivial, so $[f] \bullet\left[u_{2}\right]=0$. This concludes the proof.

Remark. Two maps $(\mathbb{D}, \partial \mathbb{D}) \rightarrow(W, \Sigma)$ that are sufficiently $C^{0}$-close are homotopic. Thus, with Proposition 8.2 we can define an intersection product on $\pi_{2}(W, \Sigma)$.

\section{Positivity of intersections of HOLOMORPhiC DisCS}

We now want to apply the topological results of the preceding section to holomorphic discs $(\mathbb{D}, \partial \mathbb{D}) \rightarrow\left(D^{4}, \tilde{S}^{t}\right)$. For future reference we formulate the results in slightly greater generality. Thus, let $(W, J)$ be a smooth almost complex 4-manifold with $J$-convex boundary $\partial W$, and $\Sigma \subset \partial W$ an embedded oriented surface totally real with respect to $J$.

In the present section, by holomorphic disc we shall always mean a smooth non-constant $J$-holomorphic disc $(\mathbb{D}, \partial \mathbb{D}) \rightarrow(W, \Sigma)$. As we saw in the previous section, any holomorphic disc is admissible. Two holomorphic discs $u_{1}, u_{2}$ are called distinct if $u_{1}(\mathbb{D}) \neq u_{2}(\mathbb{D})$.

Proposition 9.1. Any two distinct holomorphic discs intersect nicely.

Proof. By the comment after the definition of 'nicely intersecting' in the previous section, it suffices to show that any two distinct holomorphic discs intersect in finitely many points only. We are going to prove the contrapositive. That is, let $u_{1}, u_{2}$ be two holomorphic discs for which $S\left(u_{1}, u_{2}\right)$ is infinite. We have to show that $u_{1}(\mathbb{D})=u_{2}(\mathbb{D})$.

In the infinite set $S\left(u_{1}, u_{2}\right)$ we can choose a non-constant sequence $\left(z_{1}^{\nu}, z_{2}^{\nu}\right)$, $\nu \in \mathbb{N}$, converging to some point $\left(z_{1}^{0}, z_{2}^{0}\right) \in S\left(u_{1}, u_{2}\right)$. If $\left(z_{1}^{0}, z_{2}^{0}\right) \in S_{\text {Int }}\left(u_{1}, u_{2}\right)$, then by the work of Micallef-White [23, Theorem 7.1] one can find neighbourhoods $U_{i} \in \mathbb{D}$ of $z_{i}^{0}, i=1,2$, with $u_{1}\left(U_{1}\right)=u_{2}\left(U_{2}\right)$. We claim that the same conclusion holds for $\left(z_{1}^{0}, z_{2}^{0}\right) \in S_{\partial}\left(u_{1}, u_{2}\right)$. Accepting this claim for the time being, we then see that no matter where the intersection points accumulate, the set of points in $\mathbb{D}$ that have a neighbourhood mapped by $u_{1}$ into $u_{2}(\mathbb{D})$ is non-empty. This set is open by definition, and closed by the same argument used to show that it is non-empty. Hence $u_{1}(\mathbb{D}) \subset u_{2}(\mathbb{D})$. The converse inclusion holds by symmetry of the argument.

It remains to prove the claim. As in the topological situation we may choose local models such that $u_{1}, u_{2}$ around $z_{1}^{0}, z_{2}^{0}$, respectively, may be regarded as germs of maps

$$
(\mathbb{H}, \mathbb{R}, 0) \longrightarrow(\mathbb{H} \times \mathbb{C}, \mathbb{R} \times \mathbb{R}, 0)
$$

of the form

$$
u_{1}(z)=(z, 0) \text { and } u_{2}(z)=(a(z), b(z)) .
$$

Moreover, we may assume that in this local model the almost complex structure $J$ coincides with the standard structure $J_{0}$ along $\mathbb{H} \times\{0\} \subset \mathbb{H} \times \mathbb{C}$. The assumption on $\left(z_{1}^{0}, z_{2}^{0}\right)$ being an accumulation point of intersections translates into saying that $b$ has an accumulation point of zeros in $z=0$. 
The following interpolation argument is reminiscent of the proof of the unique continuation theorem in [22, p. 24]. We write

$$
J(a, b)-J(a, 0)=\int_{0}^{1} \frac{d}{d t} J(a, t b) d t=\int_{0}^{1} D_{2} J_{(a, t b)}(b) d t .
$$

This can now be used to simplify the Cauchy-Riemann equation for $u_{2}$.

$$
\begin{aligned}
0=\partial_{x} u_{2}+J\left(u_{2}\right) \partial_{y} u_{2} & =\partial_{x} u_{2}+J_{0} \partial_{y} u_{2}+\left(J\left(u_{2}\right)-J_{0}\right) \partial_{y} u_{2} \\
& =\partial_{x} u_{2}+J_{0} \partial_{y} u_{2}+(J(a, b)-J(a, 0)) \partial_{y} u_{2} \\
& =\partial_{x} u_{2}+J_{0} \partial_{y} u_{2}+\int_{0}^{1} D_{2} J_{(a, t b)}(b) d t \cdot \partial_{y} u_{2} .
\end{aligned}
$$

Define a smooth map $B=\left(B_{1}, B_{2}\right)$ from $\mathbb{H}$ into the real linear maps $\mathbb{C} \rightarrow \mathbb{C}^{2}$ by

$$
B_{z} \eta=\int_{0}^{1} D_{2} J_{(a(z), t b(z))}(\eta) d t \cdot \partial_{y} u_{2}(z) .
$$

Then the Cauchy-Riemann equation for $u_{2}$ decouples into two 1-dimensional equations

$$
\begin{aligned}
\partial_{x} a+i \partial_{y} a+B_{1} b & =0 \\
\partial_{x} b+i \partial_{y} b+B_{2} b & =0 .
\end{aligned}
$$

Now one applies a relative version of the Carleman similarity principle to $\left(\mathrm{CR}_{b}\right)$. For this principle in the absolute case see [13, Corollary 13] (for the 1-dimensional situation), [16, Section A.6] and [22, Theorem 2.3.5]. The key to a relative version of this principle is the observation (Step 2 in the proof of [22, Theorem 2.3.5]) that $B_{2}$ may be assumed to be complex linear. Then both the solution $b$ of our linear equation and the map $B_{2}$ can be extended from $\mathbb{H}$ to $\mathbb{C}$ (near 0 ) by Schwarz reflection. The Carleman similarity principle (cf. Step (v) in the proof of Proposition 7.5) then implies that the solution $b$ is a pointwise complex linear transformation of a holomorphic function. The identity theorem applied to this holomorphic function yields that this function, and hence $b$, is identically zero.

Remark. Relative versions of the Carleman similarity principle have been mentioned previously in [20, Proposition 3.1] and [1, Lemma 3.1].

In the following theorem we use $|$.$| to denote the cardinality of a finite set.$

Theorem 9.2 (Positivity of intersections). The intersection number of distinct holomorphic discs $u_{1}, u_{2}$ satisfies the inequality

$$
u_{1} \bullet u_{2} \geq 2\left|S_{\text {Int }}\left(u_{1}, u_{2}\right)\right|+\left|S_{\partial}\left(u_{1}, u_{2}\right)\right|,
$$

with equality if and only if all intersections are transverse. In particular, $u_{1}(\mathbb{D})$ and $u_{2}(\mathbb{D})$ are disjoint if and only if $u_{1} \bullet u_{2}=0$.

Proof. We need to show that the intersection multiplicities $\left(u_{1} \cdot u_{2}\right)_{\left(z_{1}, z_{2}\right)}$ are greater than or equal to 1 , with equality precisely in the case of a transverse intersection. For interior intersection points this result is due to Micallef-White [23, Theorem 7.1], cf. [22, Proposition E.2.2].

At a transverse boundary intersection point the intersection multiplicity is 1 ; this is seen exactly as for interior transverse intersections.

For a tangential boundary intersection point we use the local model from the preceding proof. Thus, write $u_{1}$ and $u_{2}$ near the intersection point $\left(z_{1}, z_{2}\right)=$ 
$(0,0) \in \mathbb{H} \times \mathbb{H}$ as $u_{1}(z)=(z, 0)$ and $u_{2}(z)=(a(z), b(z))$, where $a$ and $b$ satisfy the linear Cauchy-Riemann equations $\left(\mathrm{CR}_{a, b}\right)$ and $\left(\mathrm{CR}_{b}\right)$, respectively. The condition that the intersection point be tangential means that $d b_{0}=0$. Then $d a_{0} \neq 0$, since boundary points are non-singular. The relative Carleman similarity principle, applied to $\left(\mathrm{CR}_{b}\right)$, tells us that $b$ is of the form $b(z)=b_{k} z^{k}+o\left(\left|z^{k}\right|\right)$ with $k \geq 2$ and $0 \neq b_{k} \in \mathbb{C}$; this follows from the observation that the pointwise complex linear transformation from $b$ to a holomorphic function may be taken as the identity at $z=0$ by incorporating the transformation at 0 as a multiplicative constant into the holomorphic function.

Moreover, from $\left(\mathrm{CR}_{a}\right)$ we see with $b(0)=0$ that the differential $d a_{0}$ is complex linear. It follows that the intersection multiplicity is $k \geq 2$.

We now want to use these results to investigate the self-intersections of holomorphic discs. This will yield the criterion for a holomorphic disc to be embedded that we need for the proof of Proposition 4.5.

Definition. Let $A \in \pi_{2}(W, \Sigma)$ be a relative homotopy class, represented by a $C^{0}$. map $u:(\mathbb{D}, \partial \mathbb{D}) \rightarrow(W, \Sigma)$. By Section 8 , the intersection number $A \bullet A$ is well defined. Write $\mu(A)$ for the Maslov index of the bundle pair $\left(u^{*} T W,\left(\left.u\right|_{\partial \mathbb{D}}\right)^{*} T \Sigma\right)$ over $(\mathbb{D}, \partial \mathbb{D})$. The embedding defect of $A$ is

$$
D(A):=A \bullet A-\mu(A)+2 .
$$

The set of self-intersection points of a holomorphic disc $u$ is

$$
S(u):=\left\{\left(z_{1}, z_{2}\right) \in \mathbb{D} \times \mathbb{D}: u\left(z_{1}\right)=u\left(z_{2}\right), z_{1} \neq z_{2}\right\} .
$$

Notice that self-intersection points come in pairs $\left(z_{1}, z_{2}\right),\left(z_{2}, z_{1}\right)$. As in Section 8 we can speak of the interior and the boundary self-intersection points. Write $\operatorname{Crit}(u) \subset$ $\mathbb{D}$ for the set of critical points of $u$. Since $u$ is admissible, $\operatorname{Crit}(u)$ is contained in $\operatorname{Int} \mathbb{D}$ and does not have any accumulation points on $\partial \mathbb{D}$. Hence, Crit $(u)$ is a finite set by [22, Lemma 2.4.1], according to which critical points cannot accumulate at an interior point.

Before we can prove an estimate on the embedding defect of a holomorphic disc in terms of the disc's self-intersections and critical points, we need a formula that allows us to compute the Maslov index of a bundle pair of complex rank 1 from the self-intersection of the zero section, analogous to the formula for first Chern class of a complex line bundle, cf. [31, Proposition 2.7]. We write $\operatorname{ord}_{z} s$ for the order of an isolated zero $z$ of a continuous bundle section $s$, that is, the intersection index with the zero section.

Lemma 9.3. Let $(E, F)$ be a complex rank 1 bundle pair over $(\mathbb{D}, \partial \mathbb{D})$. Let $s$ be a continuous section of $(E, F)$ with isolated zeros. Then the Maslov index of the bundle pair is given by

$$
\mu(E, F)=2 \sum_{z \in \operatorname{Int} \mathbb{D}} \operatorname{ord}_{z} s+\sum_{z \in \partial \mathbb{D}} \operatorname{ord}_{z} s .
$$

Proof. We use a doubling argument (based on Schwarz reflection) as in [15, p. 157]. Write $\overline{\mathbb{D}}$ for the disc $\mathbb{D}$ with reversed orientation. We double $\mathbb{D}$ to the Riemann sphere $S^{2}=\mathbb{D} \cup_{\partial \mathbb{D}} \overline{\mathbb{D}}$. Reflection in $\partial \mathbb{D}$ defines an anti-holomorphic involution on $S^{2}$. 
The double of $E$ is constructed similarly. Write $\bar{E}$ for the complex line bundle over $\overline{\mathbb{D}}$ whose fibre $\bar{E}_{\bar{z}}$ over $\bar{z} \in \overline{\mathbb{D}}$ is $E_{z}$ with the conjugate complex structure. The gluing of $E_{z}$ with $\bar{E}_{\bar{z}}$ over $z \in \partial \mathbb{D}$ is effected by the anti-complex involution in $F_{z}$.

Then the section $s$ doubles to a continuous section $s \cup \bar{s}$ of the complex line bundle $E \cup \bar{E}$ over $S^{2}$, with the total order of zeros ord $(s \cup \bar{s})$ given by the righthand side of the equation in the lemma. Now the composition formula for the Maslov index [22, Theorem C.3.5] and the relation of the Maslov index with the Chern class [22, Theorem C.3.6] yield

$$
\begin{aligned}
2 \mu(E, F) & =\mu(E, F)+\mu(\bar{E}, F)=\mu(E \cup \bar{E}, \emptyset) \\
& =2\left\langle c_{1}(E \cup \bar{E}),\left[S^{2}\right]\right\rangle=2 \operatorname{ord}(s \cup \bar{s}) .
\end{aligned}
$$

The claimed formula for the Maslov index follows.

The following theorem is a quantitative version of [31, Theorem 7.6]; cf. [23, Theorem 7.3] for the adjunction inequality in the absolute case. Recall the definition of a simple disc from Section 4 .

Theorem 9.4 (Relative adjunction inequality). Let u be a simple holomorphic disc. Then the set $S(u)$ of self-intersections is finite, and the embedding defect satisfies

$$
D([u]) \geq 2\left|S_{\text {Int }}(u)\right|+\left|S_{\partial}(u)\right|+4|\operatorname{Crit}(u)| .
$$

For $\operatorname{Crit}(u)=\emptyset$ we have equality in this formula if and only if all self-intersections are transverse. In particular, $u$ is an embedding if and only if $D([u])=0$.

Proof. The finiteness of $S(u)$ is shown as in the proof of Proposition 9.1 here the assumption that $u$ be simple is used.

A lemma of Frauenfelder [9], cf. [22, Lemma 4.3.3] allows us to choose a Riemannian metric on $W$ for which $J$ is an orthogonal endomorphism field, and for which the totally real submanifold $\Sigma$ is totally geodesic.

First case: $\operatorname{Crit}(u)$ is empty. Then $(d u(T \mathbb{D}), d u(T \partial \mathbb{D}))$ is a subbundle pair of $\left(u^{*} T W,\left(\left.u\right|_{\partial \mathbb{D}}\right)^{*} T \Sigma\right)$ of Maslov index 2. By our choice of metric, the orthogonal complement of this subbundle pair is again a subbundle pair; write $\mu^{\perp}$ for its Maslov index. Then

$$
D([u])=u \bullet u-\mu^{\perp} .
$$

Choose a smooth section $s$ of this orthogonal bundle transverse to the zero section. We may assume that the zeros of $s$ are disjoint from the non-injective points of $u$, i.e. the points $z \in \mathbb{D}$ for which the preimage $u^{-1}(u(z))$ contains a point other than $z$. For $s$ sufficiently small, an admissible disc $u_{2}$ nicely intersecting $u_{1}:=u$ can be defined by $u_{2}:=\exp _{u_{1}} s$. By the homotopy invariance of the intersection number we have $u \bullet u=u_{1} \bullet u_{2}$. The intersection number $u_{1} \bullet u_{2}$ is given as a sum over intersection multiplicities, with interior intersections counting twice. Diagonal intersection points $(z, z)$ of $u_{1}$ and $u_{2}$ correspond exactly to the zeros of the section $s$. By the preceding lemma it follows that the contribution of these diagonal intersection points to $u_{1} \bullet u_{2}$ equals $\mu^{\perp}$.

We claim that the contribution of the non-diagonal intersection points to $u_{1} \bullet u_{2}$ equals the sum over the multiplicities of the self-intersection points of $u$ (with interior points counted twice). These non-diagonal intersection points arise in pairs $\left(z_{1}^{\prime}, z_{2}^{\prime}\right),\left(z_{1}^{\prime \prime}, z_{2}^{\prime \prime}\right)$ corresponding to a pair of self-intersection points $\left(z_{1}, z_{2}\right),\left(z_{2}, z_{1}\right) \in$ 
$S(u)$. So the two sums of intersection multiplicities are indeed equal. This discussion can be summarised in the formula

$$
D([u])=2 \sum_{S_{\mathrm{Int}}(u)}(u \cdot u)_{\left(z_{1}, z_{2}\right)}+\sum_{S_{\partial}(u)}(u \cdot u)_{\left(z_{1}, z_{2}\right)} .
$$

From here the argument concludes as in the proof of Theorem 9.2 ,

Second case: $\operatorname{Crit}(u)$ is non-empty. Here one can use a perturbation argument to turn critical points into self-intersections, see [22, Proposition E.2.4]. A critical point $z$ of a holomorphic disc $u$ is said to be of order $k \in \mathbb{N}$ if at $z$ the $k$-jet of $d u$ is the lowest order non-vanishing jet. For instance, a critical point of order 1 is characterised by the non-vanishing of the Hessian of $u$. As shown in [22, p. 610] and [23. Theorem 7.3], each critical point of order $k$ gives rise to at least $k(k+1)$ intersection points. Since all critical points lie in the interior, these intersection points are counted twice in the intersection product, i.e. the contribution to the embedding defect is $2 k(k+1)$.

If a critical point happens to be an intersection point of the original disc, we may think of the situation near this point as the intersection of two local discs. When we perturb and desingularise these two local discs, we obtain transverse intersections of the two local discs and self-intersections. The former contribute to the intersection product according to the intersection index, the latter contribute as analysed in the foregoing paragraph.

\section{REFERENCES}

[1] C. Abbas, Pseudoholomorphic strips in symplectisations III. Embedding properties and compactness, J. Symplectic Geom. 2 (2004), 219-260.

[2] B. Aebischer, M. Borer, M. Kälin, Ch. Leuenberger and H. M. Reimann, Symplectic Geometry, Progr. Math. 124, Birkhäuser Verlag, Basel (1994).

[3] G. E. Bredon, Topology and Geometry, Grad. Texts in Math. 139, Springer-Verlag, Berlin (1993).

[4] J. CERF, Sur les difféomorphismes de la sphère de dimension trois $\left(\Gamma_{4}=0\right)$, Lecture Notes in Math. 53, Springer-Verlag, Berlin (1968).

[5] Ya. Eliashberg, Filling by holomorphic discs and its applications, in: Geometry of LowDimensional Manifolds, Vol. 2 (Durham, 1989), London Math. Soc. Lecture Note Ser. 151, Cambridge University Press (1990), 45-67.

[6] Ya. Eliashberg, Contact 3-manifolds twenty years since J. Martinet's work, Ann. Inst. Fourier (Grenoble) 42 (1992), 165-192.

[7] Ya. Eliashberg and L. Polterovich, Local Lagrangian 2-knots are trivial, Ann. of Math. (2) 144 (1996), 61-76.

[8] L. C. Evans, Partial Differential Equations, Grad. Stud. Math. 19, American Mathematical Society, Providence, RI (1998).

[9] U. Frauenfelder, Gromov convergence of pseudoholomorphic discs, Diplomarbeit, ETH Zürich (2000).

[10] H. Geiges, An Introduction to Contact Topology, Cambridge Stud. Adv. Math. 109, Cambridge University Press, Cambridge (2008).

[11] M. Gromov, Pseudoholomorphic curves in symplectic manifolds, Invent. Math. 82 (1985), $307-347$.

[12] M. W. Hirsch, Differential Topology, Grad. Texts in Math. 33, Springer-Verlag, Berlin (1976).

[13] H. Hofer, Pseudoholomorphic curves in symplectizations with applications to the Weinstein conjecture in dimension three, Invent. Math. 114 (1993), 515-563.

[14] H. Hofer, Holomorphic curves and dynamics in dimension three, in: Symplectic Geometry and Topology (Park City, UT, 1997), IAS/Park City Math. Ser. 7, American Mathematical Society, Providence, RI (1999), 35-101. 
[15] H. Hofer, V. Lizan and J.-C. Sikorav, On genericity for holomorphic curves in fourdimensional almost-complex manifolds, J. Geom. Anal. 7 (1997), 149-159.

[16] H. Hofer And E. Zehnder, Symplectic Invariants and Hamiltonian Dynamics, Birkhäuser Adv. Texts: Basler Lehrbücher, Birkhäuser Verlag, Basel (1994).

[17] M. A. Kervaire and J. W. Milnor, Groups of homotopy spheres I, Ann. of Math. (2) 77 (1963), 504-537.

[18] A.A. Kosinski, Differential Manifolds, Academic Press, Boston, MA (1993).

[19] S. LANG, Fundamentals of Differential Geometry, Grad. Texts in Math. 191, Springer-Verlag, Berlin (1999).

[20] L. Lazzarini, Existence of a somewhere injective pseudo-holomorphic disc, Geom. Funct. Anal. 10 (2000), 829-862.

[21] D. McDuff, Singularities and positivity of intersections of $J$-holomorphic curves, in: Holomorphic Curves in Symplectic Geometry, Progr. Math. 117, Birkhäuser Verlag, Basel (1994), 191-215.

[22] D. McDuff and D. Salamon, J-holomorphic Curves and Symplectic Topology, Amer. Math. Soc. Colloq. Publ. 52, American Mathematical Society, Providence, RI (2004).

[23] M. J. Micallef And B. White, The structure of branch points in minimal surfaces and in pseudoholomorphic curves, Ann. of Math. (2) 141 (1995), 35-85.

[24] J. Munkres, Differentiable isotopies on the 2-sphere, Michigan Math. J. 7 (1960), 193-197.

[25] J. Munkres, Obstructions to imposing differentiable structures, Illinois J. Math. 8 (1964), $361-376$

[26] M. H. Protter and H. F. Weinberger, Maximum Principles in Differential Equations, Springer-Verlag, Berlin (1967).

[27] J.-C. Sikorav, Some properties of holomorphic curves in almost complex manifolds, in: Holomorphic Curves in Symplectic Geometry, Progr. Math. 117, Birkhäuser Verlag, Basel (1994), 165-189.

[28] S. Smale, Diffeomorphisms of the 2-sphere, Proc. Amer. Math. Soc. 10 (1959), 621-626.

[29] S. Smale, Generalized Poincaré's conjecture in dimensions greater than four, Ann. of Math. (2) 74 (1961), 391-406.

[30] C. Wendu, Finite Energy Foliations and Surgery on Transverse Links, Ph.D. Thesis, New York University (2005).

[31] R. Ye, Filling by holomorphic curves in symplectic 4-manifolds, Trans. Amer. Math. Soc. 350 (1998), 213-250.

[32] K. Zenmisch, The Eliashberg-Gromov Tightness Theorem, Diplomarbeit, Universität Leipzig (2003).

Mathematisches Institut, Universität Zu Köln, Weyertal 86-90, 50931 KÖln, GerMANY

E-mail address: geiges@math.uni-koeln.de, kai.zehmisch@math.uni-koeln.de 